\title{
ON THE DERIVED FUNCTORS OF DESTABILIZATION AND OF ITERATED LOOP FUNCTORS
}

\author{
GEOFFREY POWELL
}

\begin{abstract}
These notes explain how to construct small functorial chain complexes which calculate the derived functors of destabilization (respectively iterated loop functors) in the theory of modules over the mod 2 Steenrod algebra; this shows how to unify results of Singer and of Lannes and Zarati.
\end{abstract}

\section{INTRODUCTION}

These notes consider the interface between unstable modules $\mathscr{U}$ over the Steenrod algebra $\mathscr{A}$ and $\mathscr{M}$, the category of $\mathscr{A}$-modules, in particular the structure of the left derived functors of destabilization $D: \mathscr{M} \rightarrow \mathscr{U}$ (which is the left adjoint to the inclusion $\mathscr{U} \subset \mathscr{M}$ ) and of the family of iterated loop functors, $\Omega^{t}: \mathscr{U} \rightarrow \mathscr{U}$ (the left adjoint to the suspension functor $\Sigma^{t}: \mathscr{U} \rightarrow \mathscr{U}$ ), for $t \in \mathbb{N}$. For clarity of exposition, the prime 2 is privileged, and the underlying field $\mathbb{F}$ is usually the prime field $\mathbb{F}_{2}$; there are however analogous results for odd primes.

The motivation comes from the study of mod 2 singular cohomology. The Steenrod algebra $\mathscr{A}$ is the algebra of stable cohomology operations for mod 2 cohomology, hence the cohomology of a spectrum (i.e. an object from stable homotopy theory) is naturally an $\mathscr{A}$-module. The cohomology of a space has more structure: it is an unstable $\mathscr{A}$-module and is equipped with the cup product. The suspension on spectra (or pointed topological spaces) corresponds via reduced cohomology to the algebraic suspension; on $\mathscr{M}$ this is an equivalence of categories but not on unstable modules $\mathscr{U}$. The algebraic loop functor $\Omega^{t}: \mathscr{U} \rightarrow \mathscr{U}$ is a first approximation to the behaviour in cohomology of the iterated loop functor on pointed topological spaces. Similarly, the destabilization functor $D: \mathscr{M} \rightarrow \mathscr{U}$ gives a first algebraic approximation to the behaviour in cohomology of the infinite loop space functor $\Omega^{\infty}$ from spectra to pointed spaces. (This is an over-simplification, since it takes no account of the cup product; see Section 2.5 for slightly more precision.)

The two basic algebraic ingredients which are used are the Singer functors $R_{s}, s \in \mathbb{N}$, which are defined for all $\mathscr{A}$-modules, and the Singer residue map $\mathbb{F}\left[u^{ \pm 1}\right] \rightarrow \Sigma^{-1} \mathbb{F}$, which is $\mathscr{A}$-linear and induces differentials. Part of the interest of the current approach is that it provides a clear explanation of the relationship between the methods of Lannes and Zarati [LZ87] and those of Singer ([Sin81] etc.). The work of Lannes and Zarati makes no allusion to chain complexes; from the current viewpoint, they are considering the degenerate case where the differential is trivial. It is worth noting that their argument makes essential usage of connectivity, which is also a key point in the strategy used here.

This text explains how to construct a natural chain complex $\mathfrak{D} M$, for $M$ an $\mathscr{A}$ module, with homology calculating the derived functors of destabilization, and, for $t \in \mathbb{N}$ and $N$ an unstable module, a chain complex $\mathfrak{C}^{t} N$, with homology calculating

2000 Mathematics Subject Classification. Primary 55S10; Secondary 18E10.

Key words and phrases. Steenrod algebra - unstable module - destabilization - iterated loop functor - derived functor - total Steenrod power. 
G. POWELL

the derived functors of $\Omega^{t}$. The existence of such a chain complex goes back to the work of Singer [Sin78, Sin80, but the construction given here is new.

The complex $\mathfrak{C}^{t} N$ is given as a quotient of $\mathfrak{D}\left(\Sigma^{-t} N\right)$ and the projection

$$
\mathfrak{D}\left(\Sigma^{-t} N\right) \rightarrow \mathfrak{C}^{t} N
$$

induces in homology the natural transformation $D_{s}\left(\Sigma^{-t} N\right) \rightarrow \Omega_{s}^{t} N$ between left derived functors.

The chain complex $\mathfrak{D} M$ is also related to the chain complex $\Gamma^{+} M$ introduced by Singer [Sin83] and Nguyễn H. V. Hưng and Nguyễn Sum HS95] (who work at odd primes), to calculate the homology of $M$ over the Steenrod algebra. Namely, there is a natural inclusion

$$
\mathfrak{D} M \hookrightarrow \Gamma^{+} M
$$

which, in homology, induces the Lannes-Zarati homomorphism (up to dualizing) [LZ87, the derived form of:

$$
D M \rightarrow \mathbb{F} \otimes \mathscr{A} M
$$

thus giving rise to $D_{s} M \rightarrow \operatorname{Tor}_{s}^{\mathscr{A}}(\mathbb{F}, M)$. (A word of warning: $D M$ is an unstable module, in particular concentrated in degrees $\geq 0$, hence the map to $\mathbb{F} \otimes \mathscr{A} M$ is not in general surjective.) This morphism is of interest, since it is intimately related to the mod 2 Hurewicz morphism.

The final section indicates some recent developments in the subject and some open problems. In particular, the potential higher chromatic analogues of this theory are of significant interest. This material is only outlined here.

A number of exercises (of varying levels of difficulty) are included, reflecting the origin of this text as lecture notes; likewise, some proofs are left as exercises. The reader is encouraged to attempt them all, since they are essential to the understanding of the subject.

Acknowledgement: The author is grateful to the anonymous referee for their careful reading of the manuscript and for their suggestions.

The author would like to thank the VIASM for the invitation to give this lecture course and for providing excellent working conditions during his visit in August 2013. He is also grateful to the participants of the special activity on Algebraic Topology for their interest.

Finally, the author also wishes to acknowledge the financial support provided by the VIASM and LIA Formath.

\section{Contents}

1. Introduction

2. Background

3. First results on derived functors of destabilization and of iterated loops 10

4. Singer functors

5. Constructing chain complexes

6. Perspectives

References 


\section{BACKGROUND}

Throughout, unless indicated otherwise, the underlying prime is taken to be 2 and the ground field $\mathbb{F}$ is the field $\mathbb{F}_{2}$ with two elements. All the results introduced have analogues for odd primes, although the arguments are slightly more complicated in the odd primary situation.

A general reference for the theory of (unstable) modules over the Steenrod algebra is the book by Schwartz [Sch94] and, for $\mathscr{A}$-modules, that of Margolis [Mar83]. References for the results stated can be found for example in the author's papers Pow10, Pow14, Pow12; many go back to Massey and Peterson and the work of Singer.

2.1. The Steenrod algebra as a quadratic algebra. The mod 2 Steenrod algebra $\mathscr{A}$ is, by definition, the algebra of stable cohomology operations for mod 2 singular cohomology. Hence the Steenrod algebra can be identified with the cohomology $H^{*}\left(H \mathbb{F}_{2}\right)$ (here $H^{*}(-)$ always denote cohomology with mod 2 coefficients) of the Eilenberg-MacLane spectrum, $H \mathbb{F}_{2}$, which represents $\bmod 2$ cohomology.

The algebra $\mathscr{A}$ is a non-homogeneous quadratic algebra, as explained below. Let $\tilde{\mathscr{A}}$ be the algebra

$$
\tilde{\mathscr{A}}:=T\left(S q^{i} \mid i \geq 0\right) / \sim
$$

where $S q^{i}$ has degree $i$ and $\sim$ corresponds to the Adem relations (for $a<2 b$ ):

$$
S q^{a} S q^{b}=\sum_{j}^{[a / 2]}\left(\begin{array}{c}
b-j-1 \\
a-2 j
\end{array}\right) S q^{a+b-j} S q^{j},
$$

where $S q^{0}$ is considered as an independent generator. Since the relations are homogeneous of length 2 , the algebra $\tilde{\mathscr{A}}$ is a homogeneous quadratic algebra and, in particular, has a length grading in addition to the internal grading coming from the degrees of the generators.

There is a surjection of algebras $\tilde{\mathscr{A}} \rightarrow \mathscr{A}$ which corresponds to imposing the relation $S q^{0}=1$; the algebra $\mathscr{A}$ inherits a length filtration from $\tilde{\mathscr{A}}$ (no longer a grading). The relations defining $\mathscr{A}$ have length $\leq 2$, which means that $\mathscr{A}$ is quadratic.

The graded $\overline{\mathscr{A}}$ associated to the length filtration can also be described as a quotient of $\tilde{\mathscr{A}}$, namely

$$
\overline{\mathscr{A}}=\tilde{\mathscr{A}} /\left\langle S q^{0}\right\rangle \text {. }
$$

This is again a homogeneous quadratic algebra. Moreover, it has the important property that it is Koszul. This notion, introduced by Priddy Pri70, is at the origin of the existence of small resolutions for calculating the homology of the Steenrod algebra; the Koszul dual is the (big) Lambda algebra.

The construction of the complexes introduced here is related to the quadratic Koszul nature of $\mathscr{A}$ and also to the relationship between the Steenrod algebra and invariant theory; many of the ideas go back to the work of Singer Sin78, Sin80, Sin83 etc.

Remark 2.1.1. The odd primary analogues depend upon the work of Mùi Mùi86, Mùi75, which describes the (more complicated) relationship between invariant theory and the Steenrod algebra.

See for example the work of Nguyễn H. V. Hưng and Nguyễn Sum [HS95] generalizing Singer's invariant-theoretic description of the Lambda algebra to odd primes, Zarati's generalization [Zar84 of his work with Lannes [LZ87] and the author's paper Pow14. 
G. POWELL

2.2. The category of $\mathscr{A}$-modules. Let $\mathscr{M}$ denote the category of (left) $\mathscr{A}$ modules. This is an abelian category with additional structure; namely, the fact that $\mathscr{A}$ is a Hopf algebra implies that the tensor product (as graded vector spaces) of two $\mathscr{A}$-modules has a natural $\mathscr{A}$-module structure. Explicitly, the Steenrod squares act via:

$$
S q^{n}(x \otimes y)=\sum_{i+j=n} S q^{i}(x) \otimes S q^{j}(y)
$$

this corresponds to the fact that the diagonal $\Delta: \mathscr{A} \rightarrow \mathscr{A} \otimes \mathscr{A}$ is determined by $\Delta S q^{n}=\sum_{i+j=n} S q^{i} \otimes S q^{j}$.

Since $\mathscr{A}$ is a connected algebra (concentrated in non-negative degrees, with $\mathscr{A}^{0}=$ $\mathbb{F}$ ) the Hopf algebra conjugation (or antipode) $\chi: \mathscr{A}^{\circ} \rightarrow \mathscr{A}$ is determined by the diagonal [MM65] and is an isomorphism of algebras, where $\mathscr{A}^{\circ}$ is $\mathscr{A}$ equipped with the opposite algebra structure ( $\chi$ is an anti-automorphism of $\mathscr{A}$ ).

Via $\chi$, the category of left $\mathscr{A}$-modules is equivalent to the category of right $\mathscr{A}$ modules: a right $\mathscr{A}$-module $M$ can be considered as a left $\mathscr{A}$-module by setting $a m:=m \chi(a)$ for $a \in \mathscr{A}$ and $m \in M$.

Hence the category $\mathscr{M}$ has a duality functor:

$$
\begin{aligned}
(-)^{\vee}: \mathscr{M}^{\mathrm{op}} & \rightarrow \mathscr{M} \\
M & \mapsto M^{\vee}:=\operatorname{Hom}_{\mathbb{F}}(M, \mathbb{F}),
\end{aligned}
$$

where the usual right $\mathscr{A}$-module structure on $M^{\vee}$ is regarded as a left structure via $\chi$.

Notation 2.2.1. For $n \in \mathbb{Z}$, let $\Sigma^{n} \mathbb{F}$ denote the $\mathscr{A}$-module $\mathbb{F}$ in degree $n$.

Remark 2.2.2. Since $\mathscr{A}$ is connected, $\left\{\Sigma^{n} \mathbb{F} \mid n \in \mathbb{Z}\right\}$ gives a set of representatives of isomorphism classes of the simple objects of $\mathscr{M}$.

Example 2.2.3. Duality gives $\left(\Sigma^{n} \mathbb{F}\right)^{\vee}=\Sigma^{-n} \mathbb{F}$.

Definition 2.2.4. For $n \in \mathbb{Z}$, the $n$th suspension functor $\Sigma^{n}: \mathscr{M} \rightarrow \mathscr{M}$ is $\Sigma^{n} \mathbb{F} \otimes-$.

Proposition 2.2.5.

(1) The category $\mathscr{M}$ has enough projectives, with set of projective generators $\left\{\Sigma^{n} \mathscr{A} \mid n \in \mathbb{Z}\right\}$.

(2) For $n \in \mathbb{Z}, \Sigma^{n}: \mathscr{M} \rightarrow \mathscr{M}$ is an exact functor which is an equivalence of categories, with inverse $\Sigma^{-n}: \mathscr{M} \rightarrow \mathscr{M}$. In particular, $\Sigma^{n}$ preserves projectives.

Proof. For the first point, $\Sigma^{n} \mathscr{A}$ is a free $\mathscr{A}$-module, hence projective (in fact, $\Sigma^{n} \mathscr{A}$ is the projective cover of $\left.\Sigma^{n} \mathbb{F}\right)$. Moreover, for $M$ an $\mathscr{A}$-module, $\operatorname{Hom}_{\mathscr{M}}\left(\Sigma^{n} \mathscr{A}, M\right) \cong$ $M^{n}$, whence it follows that $\left\{\Sigma^{n} \mathscr{A} \mid n \in \mathbb{Z}\right\}$ is a set of projective generators.

The second statement is clear.

2.3. Unstable modules and destabilization. Whereas the cohomology of a spectrum (object from stable homotopy theory which represents a cohomology theory) is simply an $\mathscr{A}$-module, the cohomology of a space has further structure; it is an algebra (via the cup product) and the underlying $\mathscr{A}$-module is unstable.

Definition 2.3.1. An $\mathscr{A}$-module $M$ is unstable if $S q^{i} x=0, \forall i>|x|$. The full subcategory of unstable modules is denoted $\mathscr{U} \subset \mathscr{M}$.

Proposition 2.3.2. The category $\mathscr{U}$ is an abelian subcategory of $\mathscr{M}$ and is closed under the tensor product $\otimes$ of $\mathscr{M}$. 
Proof. From the definition of instability, it is clear that a submodule (respectively quotient) of an unstable module is unstable. This implies that $\mathscr{U}$ is an abelian subcategory of $\mathscr{M}$.

Closure under $\otimes$ is seen as follows. By definition, $S q^{n}(x \otimes y)=\sum_{i+j=n} S q^{i}(x) \otimes$ $S q^{j}(y)$; if $n>|x \otimes y|$ and $i+j=n$, then either $i>|x|$ or $j>|y|$, so that the right hand expression is zero, as required.

Remark 2.3.3. The duality functor $(-)^{\vee}: \mathscr{M}^{\text {op }} \rightarrow \mathscr{M}$ does not preserve $\mathscr{U}$, since the relation $S q^{0}=1$ implies that an unstable module is concentrated in degrees $\geq 0$. The dual $M^{\vee}$ of a module $M$ concentrated in degrees $\geq 0$ is concentrated in degrees $\geq 0$ if and only if $M=M^{0}$; for example, the dual of $\Sigma \mathbb{F}$ is not unstable.

Example 2.3.4. For $n \in \mathbb{N}$, the suspension functor $\Sigma^{n}: \mathscr{M} \rightarrow \mathscr{M}$ restricts to an exact functor $\Sigma^{n}: \mathscr{U} \rightarrow \mathscr{U}$ (given by $\Sigma^{n} \mathbb{F} \otimes-$ ). This is not an equivalence of categories if $n>0$.

For later use, the following definition is recalled, which uses the tensor product of $\mathscr{U}$.

Definition 2.3.5. An algebra in $\mathscr{M}$ is a graded algebra such that the structure morphisms are $\mathscr{A}$-linear. An unstable algebra $K$ is an unstable module which is a commutative algebra in $\mathscr{M}$ (and hence in $\mathscr{U}$ ) such that the Cartan condition holds: $S q^{|x|}(x)=x^{2}, \forall x \in K$. Unstable algebras form a category $\mathscr{K}$, with morphisms the algebra morphisms which are $\mathscr{A}$-linear. Forgetting the algebra structure yields a functor $\mathscr{K} \rightarrow \mathscr{U}$.

In a few places the terminology nilpotent, reduced, nil-closed will be used; for the convenience of the reader, the definition is recalled (see [Sch94] for further details).

Definition 2.3.6. An unstable module $N$ is nilpotent if the operation $S q_{0}$ (where $\left.S q_{0}(x):=S q^{|x|}(x)\right)$ acts locally nilpotently.

An unstable module $M$ is reduced if $\operatorname{Hom}_{\mathscr{U}}(N, M)=0$ for any nilpotent module $N$ and nil-closed if, in addition, $\operatorname{Ext}_{\mathscr{U}}^{1}(N, M)=0$ for all nilpotents $N$.

Remark 2.3.7. If $M$ is reduced, there is a nil-closed unstable module $\bar{M}$ and an inclusion $M \hookrightarrow \bar{M}$ with nilpotent cokernel. The module $\bar{M}$ is unique up to isomorphism and is called the nil-closure of $M$.

Such considerations arise when considering the localization of $\mathscr{U}$ away from the nilpotent unstable modules, namely the study of the quotient category $\mathscr{U} / \mathscr{N} i l$ (see [Sch94, Chapter 5]).

The following gives the archetypal examples of nilpotent unstable modules:

Example 2.3.8. For $M$ an unstable module, $\Sigma M$ is nilpotent.

The notion of destabilization arises naturally through topological considerations, for example when passing from stable homotopy theory (spectra) to unstable homotopy theory (spaces).

Definition 2.3.9. Let $D: \mathscr{M} \rightarrow \mathscr{U}$ be the left adjoint to the (exact) inclusion functor $\mathscr{U} \hookrightarrow \mathscr{M}$.

Exercise 2.3.10. For $M$ an $\mathscr{A}$-module, show that the linear subspace

$$
B M:=\left\langle S q^{i}(x)|i>| x \mid\right\rangle,
$$

as $x$ ranges over elements of $M$, is a sub $\mathscr{A}$-module. Deduce that $D M \cong M / B M$ is an $\mathscr{A}$-module (unstable, by construction). 
Namely, from the explicit construction, if $f: M \rightarrow N$ is a morphism of $\mathscr{A}$ modules with $N$ unstable, there is a natural factorization:

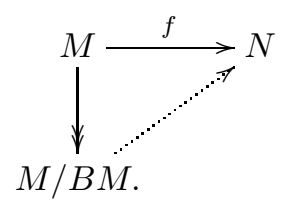

Notation 2.3.11. For $n \in \mathbb{Z}$, let $F(n)$ denote $D\left(\Sigma^{n} \mathscr{A}\right)$; this is the free unstable module on a generator of degree $n$.

Proposition 2.3.12. The category $\mathscr{U}$ has enough projectives and $\{F(n) \mid n \in \mathbb{N}\}$ forms a set of projective generators.

Proof. By adjunction, for $N$ an unstable module, there are natural isomorphisms

$$
\operatorname{Hom}_{\mathscr{U}}\left(D\left(\Sigma^{n} \mathscr{A}\right), N\right) \cong \operatorname{Hom}_{\mathscr{M}}\left(\Sigma^{n} \mathscr{A}, N\right) \cong N^{n},
$$

which show both that $D\left(\Sigma^{n} \mathscr{A}\right)$ is projective and that these form a set of projective generators (recalling that an unstable module is necessarily concentrated in nonnegative degrees).

Proposition 2.3.13. The functor $D: \mathscr{M} \rightarrow \mathscr{U}$ is right exact (but not exact) and preserves projectives.

Proof. The functor $D$ is left adjoint to the exact forgetful functor $\mathscr{M} \rightarrow \mathscr{U}$, hence preserves projectives and is right exact.

To see that $D$ is not exact, consider the $\mathscr{A}$-module $\mathscr{E}:=\Sigma^{-1} \tilde{H}^{*}\left(\mathbb{R} P^{2}\right)$. The latter lies in the non-split short exact sequence in $\mathscr{M}$ :

$$
0 \rightarrow \Sigma \mathbb{F} \rightarrow \mathscr{E} \rightarrow \mathbb{F} \rightarrow 0
$$

where the classes are linked by $S q^{1}$. Clearly $D \mathscr{E}=\mathbb{F}$ and $D \Sigma \mathbb{F}=\Sigma \mathbb{F}$, so applying $D$ exhibits the non-exactitude.

The functor $D$ can be used to define division functors. The most important examples considered here are the (iterated) loop functors.

Definition 2.3.14. For $n \in \mathbb{N}$, let $\Omega^{n}: \mathscr{U} \rightarrow \mathscr{U}$ denote the composite functor $D \Sigma^{-n}$, where $\Sigma^{-n}: \mathscr{M} \rightarrow \mathscr{M}$ is restricted to a functor $\mathscr{U} \rightarrow \mathscr{M}$. (For $n=1, \Omega^{1}$ is denoted simply $\Omega$.)

Proposition 2.3.15. For $n \in \mathbb{N}$, the functor $\Omega^{n}: \mathscr{U} \rightarrow \mathscr{U}$ is left adjoint to $\Sigma^{n}: \mathscr{U} \rightarrow \mathscr{U}$; it is right exact (but not exact for $n>0$ ) and preserves projectives.

Proof. It is straightforward to check that $D \Sigma^{-n}$ is left adjoint to $\Sigma^{n}: \mathscr{U} \rightarrow \mathscr{U}$. Since the latter is exact, it follows that $\Omega^{n}$ is right exact and preserves projectives. Non-exactitude can be seen as in Proposition 2.3.13 for example, consider applying the functor $\Omega$ to $\Sigma \mathscr{E}$ for $n=1$ (noting that $\mathscr{E}$ is not unstable).

Exercise 2.3.16. Show that, for $0<n \in \mathbb{N}, \Omega F(n) \cong F(n-1)$.

Proposition 2.3.17. For $n \in \mathbb{N}$ there is a natural equivalence of functors

$$
\Omega^{n} D \cong D \Sigma^{-n}: \mathscr{M} \rightarrow \mathscr{U} .
$$

Proof. For $N$ an unstable module and $M$ an $\mathscr{A}$-module, there is a chain of natural adjunction isomorphisms

$$
\begin{aligned}
\operatorname{Hom}_{\mathscr{U}}\left(\Omega^{n} D M, N\right) & \cong \operatorname{Hom}_{\mathscr{U}}\left(D M, \Sigma^{n} N\right) \cong \operatorname{Hom}_{\mathscr{M}}\left(M, \Sigma^{n} N\right) \\
& \cong \operatorname{Hom}_{\mathscr{M}}\left(\Sigma^{-n} M, N\right) \cong \operatorname{Hom}_{\mathscr{U}}\left(D \Sigma^{-n} M, N\right),
\end{aligned}
$$

from which the result follows. 
Exercise 2.3.18. Let $M$ be an unstable module which is of finite type (i.e. $\operatorname{dim}\left(M^{n}\right)$ is finite $\forall n)$. Show that the functor $D\left(-\otimes M^{\vee}\right)$ is left adjoint to $M \otimes-$.

This left adjoint is usually referred to as the division functor by $M$ and written $(-: M)$; see Lan92 for general considerations on such functors.

Example 2.3.19. An important division functor which can be constructed by using destabilization is Lannes' $T$-functor $T:=\left(-: H^{*}(B \mathbb{Z} / 2)\right)$, so that, for $M$ an unstable module,

$$
T M=D\left(M \otimes H^{*}(B \mathbb{Z} / 2)^{\vee}\right),
$$

where $B \mathbb{Z} / 2$ is the classifying space of the group $\mathbb{Z} / 2$, which has the homotopy type of $\mathbb{R} P^{\infty}$. (For the structure of $H^{*}(B \mathbb{Z} / 2)$, see Example 2.4.4 below.) The dual $H^{*}(B \mathbb{Z} / 2)^{\vee}$ can be identified as the homology $H_{*}(B \mathbb{Z} / 2)$, considered as a left $\mathscr{A}$-module via the conjugation $\chi$.

Remark 2.3.20. Lannes' $T$-functor is an essential tool in modern homotopy theory and is defined for any prime $p$. It has good properties which make it accessible to calculation; for example it is exact and commutes with tensor products. Moreover, the $T$-functor restricts to a functor on the category $\mathscr{K}$ of unstable algebras. An underlying fundamental algebraic fact is that the cohomology of an elementary abelian $p$-group, $H^{*}\left(B V ; \mathbb{F}_{p}\right)$, is injective in $\mathscr{U}$.

The homotopical importance of $T$ stems from the fact that $T H^{*}(X)$ is an approximation to the calculation of $H^{*}(\operatorname{Map}(B \mathbb{Z} / p, X))$. Indeed, there is a canonical comparison map $T H^{*}(X) \rightarrow H^{*}(\operatorname{Map}(B \mathbb{Z} / p, X))$ that is given by adjunction from the morphism induced in cohomology by the evaluation map

$$
\operatorname{Map}(B \mathbb{Z} / p, X) \times B \mathbb{Z} / p \rightarrow X .
$$

Under adequate hypotheses upon $X$, the comparison map is an isomorphism.

The reader should consult Sch94 for this and much more, in particular the application of $T$-functor technology to Sullivan's fixed point set conjecture (see also Lan92 ).

2.4. Derived functors. The abelian categories $\mathscr{M}$ and $\mathscr{U}$ both have enough projectives (by Propositions 2.2.5 and 2.3.12), hence one can do homological algebra in them. Recall that a projective resolution $P \bullet$ of an object $M$ of an abelian category is a complex of projectives

$$
\ldots \rightarrow P_{s} \rightarrow P_{s-1} \rightarrow \ldots \rightarrow P_{1} \rightarrow P_{0},
$$

with $P_{s}$ in homological degree $s$, and which has homology concentrated in degree zero with $H_{0}\left(P_{\bullet}\right) \cong M$. This will frequently be denoted by $P_{\bullet} \rightarrow M$, where the arrow corresponds to the surjection $P_{0} \rightarrow M$.

Remark 2.4.1. If $M$ is an unstable module, there are two possible notions of projective resolution: a projective resolution in $\mathscr{U}, P_{\bullet} \rightarrow M$ (that is, by projectives in $\mathscr{U})$, or a resolution in $\mathscr{M}, F_{\bullet} \rightarrow M$, by free $\mathscr{A}$-modules.

Definition 2.4.2. For $s, n \in \mathbb{N}$, let

(1) $D_{s}: \mathscr{M} \rightarrow \mathscr{U}$ denote the $s$ th left derived functor of $D: \mathscr{M} \rightarrow \mathscr{U}$;

(2) $\Omega_{s}^{n}: \mathscr{U} \rightarrow \mathscr{U}$ denote the $s$ th left derived functor of $\Omega^{n}: \mathscr{U} \rightarrow \mathscr{U}$.

Explicitly, if $F_{\bullet} \rightarrow M$ is a free resolution in $\mathscr{M}$ of an $\mathscr{A}$-module $M$, then $D_{s} M$ is the $s$ th homology of the complex $D F_{\bullet}$. Note that $D F_{\bullet}$ is a complex with each object $D F_{s}$ projective in $\mathscr{U}$, by Proposition 2.3.13, it is a projective resolution of $D M$ if and only if all the higher derived functors $D_{s} M$ vanish. 
G. POWELL

Exercise 2.4.3. Let $M$ be an $\mathscr{A}$-module and suppose that there exists $t \in \mathbb{N}$ such that $\Sigma^{t} M$ is unstable (such a $t$ does not exist in general - see Remark 2.4.5 below). Show that, for all $s \in \mathbb{N}$, there exists a natural morphism $D_{s} M \rightarrow \Omega_{s}^{t} \Sigma^{t} M$. This exhibits the close relationship between derived functors of destabilization and of iterated loop functors.

Example 2.4.4. Derived functors of destabilization are highly non-trivial. For example we consider a lower bound for $D_{1}\left(\Sigma^{-1} \mathbb{F}\right)$ as follows.

Recall that $H^{*}(B \mathbb{Z} / 2) \cong \mathbb{F}[u]$, where $|u|=1$; this is an unstable algebra, and this fact determines its structure as an $\mathscr{A}$-module. (Explicitly, the total Steenrod power $S q^{\mathrm{T}}=\sum_{i \in \mathbb{N}} S q^{i}$ on $u$ is $S q^{\mathrm{T}}(u)=u(1+u)$, via the Cartan condition and instability and this determines the structure via the Cartan formula for cup products, which implies that $S q^{\mathrm{T}}$ is multiplicative.)

One can form the localization $\mathbb{F}\left[u^{ \pm 1}\right]$, so that $u^{-1}$ is a class of degree -1 . This has an $\mathscr{A}$-algebra structure (not unstable!), which is determined by the total Steenrod power of $u^{-1}$. This can be calculated by using the multiplicativity of $S q^{\mathrm{T}}$ :

$$
1=S q^{\mathrm{T}}(1)=S q^{\mathrm{T}}\left(u^{-1} u\right)=S q^{\mathrm{T}}\left(u^{-1}\right) S q^{\mathrm{T}}(u),
$$

giving $S q^{\mathrm{T}}\left(u^{-1}\right)=\frac{u^{-1}}{1+u}$, which translates as

$$
S q^{n+1}\left(u^{-1}\right)=u^{n}
$$

for all $n \in \mathbb{N}$.

Let $\hat{P}$ denote the sub $\mathbb{F}[u]$-module of $\mathbb{F}\left[u^{ \pm 1}\right]$ generated by $u^{-1}$, so that there is a (non-split) short exact sequence of $\mathscr{A}$-modules:

$$
0 \rightarrow \mathbb{F}[u] \rightarrow \hat{P} \rightarrow \Sigma^{-1} \mathbb{F} \rightarrow 0 .
$$

It is straightforward to see that $D \hat{P}=0$ and $D \Sigma^{-1} \mathbb{F}=0$. Hence the long exact sequence for derived functors

$$
\ldots \rightarrow D_{1} \hat{P} \rightarrow D_{1}\left(\Sigma^{-1} \mathbb{F}\right) \rightarrow D \mathbb{F}[u] \rightarrow D \hat{P} \rightarrow D \Sigma^{-1} \mathbb{F} \rightarrow 0
$$

shows that $D_{1}\left(\Sigma^{-1} \mathbb{F}\right) \rightarrow D \mathbb{F}[u] \cong \mathbb{F}[u]$ is surjective. (It is in fact an isomorphism, by Corollary [5.1.10, ) Thus $D_{1}\left(\Sigma^{-1} \mathbb{F}\right)$ is infinite, even though $\Sigma^{-1} \mathbb{F}$ has total dimension one.

Remark 2.4.5. The $\mathscr{A}$-module $\hat{P}$ is bounded below; however $\Sigma^{t} \hat{P}$ is never unstable, since all the $S q^{i}$ act non-trivially upon the lowest dimensional class.

2.5. Motivation for studying derived functors of destabilization and of iterated loop functors. The functors $D_{s}: \mathscr{M} \rightarrow \mathscr{U}$ arise naturally in algebraic topology.

Example 2.5.1. There is a Grothendieck spectral sequence calculating $\operatorname{Ext}_{\mathscr{A}}^{*}(M, N)$ in terms of Ext $\mathscr{U}$ when $N$ is an unstable module. This is the spectral sequence derived from considering $\operatorname{Hom}_{\mathscr{A}}(-, N)$ as the composite functor $\operatorname{Hom}_{\mathscr{U}}(D(-), N)$.

The spectral sequence has the form

$$
\operatorname{Ext}_{\mathscr{U}}^{p}\left(D_{q} M, N\right) \Rightarrow \operatorname{Ext}_{\mathscr{A}}^{p+q}(M, N) .
$$

When $N$ is injective in $\mathscr{U}$ (for example $N=\mathbb{F}$ or $N=H^{*}(B V)$, for $V$ an elementary abelian 2-group), the spectral sequence degenerates to the isomorphism

$$
\operatorname{Ext}_{\mathscr{A}}^{q}(M, N) \cong \operatorname{Hom}_{\mathscr{U}}\left(D_{q} M, N\right) .
$$

Such Ext groups are important for calculating the $E^{2}$-term of the Adams spectral sequence. This motivated Lannes and Zarati's work on the derived functors of destabilization [LZ87 and is intimately related to an approach to the Segal conjecture for elementary abelian $p$-groups. 
Example 2.5.2. Derived functors of destabilization occur in studying the relationship between the cohomology of a spectrum $E$ and the cohomology of the infinite loop space $\Omega^{\infty} E$ associated to $E$.

Recall that there is an adjunction counit $\Sigma^{\infty} \Omega^{\infty} E \rightarrow E$, where $\Sigma^{\infty}$ is the suspension spectrum functor. This gives rise to a commutative diagram in $\mathscr{M}$ :

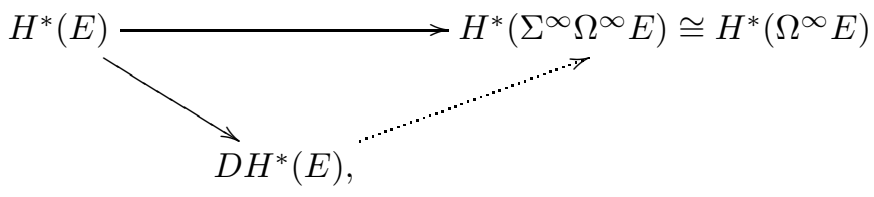

where the factorization exists since $H^{*}\left(\Omega^{\infty} E\right)$ is an unstable algebra and, in particular, an unstable module.

Recall from Definition 2.3.5 that $\mathscr{K}$ denotes the category of unstable algebras and that the Steenrod-Epstein enveloping algebra functor $U: \mathscr{U} \rightarrow \mathscr{K}$ is left adjoint to the forgetful functor $\mathscr{K} \rightarrow \mathscr{U}$. This is given explicitly by

$$
U M:=S^{*}(M) /\left\langle S q^{|m|} m=m^{2}\right\rangle,
$$

the quotient of the free commutative algebra on $M$ given by imposing the Cartan condition.

Hence, the above induces a morphism of unstable algebras:

$$
U\left(D H^{*}(E)\right) \rightarrow H^{*}\left(\Omega^{\infty} E\right) .
$$

When $E=\Sigma^{n} H \mathbb{F}_{2}$ is a suspension of the mod 2 Eilenberg-MacLane spectrum, this is an isomorphism. However, in general it is far from being an isomorphism (examples can be given by considering suspension spectra $E=\Sigma^{\infty} X$ ). Haugseng and Miller [HM16] have constructed a spectral sequence which, in favourable circumstances, calculates $H^{*}\left(\Omega^{\infty} E\right)$ from $H^{*}(E)$,

based on a cosimplicial Adams resolution of $E$ constructed from EilenbergMacLane spectra. The $E_{2}$-term is expressed in terms of non-abelian derived functors of $U D$. In particular, they show how the derived functors of destabilization intervene.

Kuhn and McCarty KM13 construct a spectral sequence to calculate $H^{*}\left(\Omega^{\infty} E\right)$ using the Goodwillie-Arone tower associated to the functor $E \mapsto \Sigma^{\infty} \Omega^{\infty} E$. In addition, they exhibited an algebraic approximation to $H^{*}\left(\Omega^{\infty} E\right)$, which is expressed in terms of the derived functors of destabilization. This generalizes earlier work of Lannes and Zarati [LZ84 for suspension spectra.

Example 2.5.3. Similar considerations arise in giving an algebraic approximation to $H^{*}\left(\Omega^{n} X\right)$ in terms of $H^{*}(X)$, when $X$ is a pointed space. As a first approximation, one shows that there is a natural morphism of unstable algebras

$$
U\left(\Omega^{n} Q H^{*}(X)\right) \rightarrow H^{*}\left(\Omega^{n} X\right),
$$

where $Q: \mathscr{K}_{a} \rightarrow \mathscr{U}$ is the 'indecomposables' functor, defined on the category $\mathscr{K}_{a}$ of augmented unstable algebras by $Q K:=\bar{K} / \bar{K}^{2}$, where $\bar{K}$ is the augmentation ideal. (The base point of $X$ induces the augmentation of $H^{*}(X)$.) This can be shown to be an isomorphism for Eilenberg-MacLane spaces but, in general, is far from being an isomorphism.

Under suitable hypotheses on the space $X$, in particular supposing that the cohomology of $X$ is of the form $U M$ for some unstable module $M$, Harper and Miller HM89 gave an algebraic approximation to $H^{*}\left(\Omega^{n} X\right)$, which is expressed in terms of the derived functors of certain iterated loop functors. It is expected that their results can be generalized. 
G. POWELL

Remark 2.5.4. Note that the topological based loop space functor $\Omega$ is right adjoint to the reduced suspension functor $\Sigma$ and the infinite loop space functor $\Omega^{\infty}$ is right adjoint to the suspension spectrum functor $\Sigma^{\infty}$ (at the level of homotopy categories). The suspension functor $\Sigma$ commutes with cohomology; however, since $H^{*}(-)$ is contravariant, the algebraic approximations to these functors are left adjoints.

\section{First RESUlts ON DERIVED FUnCTORS OF DESTABILIZATION AND OF ITERATED LOOPS}

In this section, elementary results on the derived functors of $D$ and $\Omega^{n}$ are considered, as a warm-up to constructing the chain complexes which compute the respective derived functors.

3.1. Derived functors of $\Omega$. There is a simple chain complex which calculates the derived functors of $\Omega$; to define it requires the introduction of the doubling (or Frobenius) functor $\Phi$.

Definition 3.1.1. Let $\Phi: \mathscr{M} \rightarrow \mathscr{M}$ be the functor which associates to $M$ the module $\Phi M$ concentrated in even degrees with $(\Phi M)^{2 k}=M^{k}$ and action of the Steenrod algebra determined by $S q^{2 i}(\Phi x)=\Phi\left(S q^{i} x\right)$.

Let $\lambda_{M}: \Phi M \rightarrow M$ be the natural morphism (of graded vector spaces) defined by $\lambda_{M}(\Phi x)=S q_{0}(x):=S q^{|x|}(x)$.

Remark 3.1.2. The functor $\Phi$ and the linear transformation $\lambda$ are defined for all $\mathscr{A}$-modules.

Proposition 3.1.3. If $M$ is an unstable module, $\lambda_{M}: \Phi M \rightarrow M$ is $\mathscr{A}$-linear; hence $\lambda$ induces a natural transformation $\lambda:\left.\Phi\right|_{\mathscr{U}} \rightarrow 1_{\mathscr{U}}$.

Proof. This is an important exercise in using the Adem relation for $S q^{a} S q^{|x|}(x)$ and the instability condition. By the latter, one may reduce to the case $a \leq 2|x|$; it remains to show that the right hand side is zero for $a$ odd whereas, for $a=2 j \leq 2|x|$,

$$
S q^{2 j} S q_{0}(x)=S q_{0} S q^{j}(x) .
$$

The reader should provide the details for themselves.

Remark 3.1.4. For $M$ an unstable module, $\lambda_{M}$ is injective if and only if $M$ is a reduced unstable module.

Proposition 3.1.5. The functor $\Phi: \mathscr{M} \rightarrow \mathscr{M}$ satisfies the following properties:

(1) $\Phi$ is exact;

(2) $\Phi$ commutes with tensor products, in particular $\Phi(\Sigma M) \cong \Sigma^{2} \Phi M$.

Proof. This follows directly from the definitions.

Remark 3.1.6. In odd characteristic $p$, the corresponding functor $\Phi$ does not commute with tensor products; behaviour of $\Phi \Sigma$ is complicated, whereas $\Phi \Sigma^{2} \cong \Sigma^{2 p} \Phi$.

Exercise 3.1.7. For $K$ an unstable algebra, show that $\lambda_{K}: \Phi K \rightarrow K$ is a morphism of unstable algebras. If $K$ is reduced (equivalently has no nilpotent elements as a commutative algebra), show that $\Phi K$ identifies with the subalgebra of $K$ generated by the squares of elements of $K$. For example $\Phi \mathbb{F}[u] \cong \mathbb{F}\left[u^{2}\right] \subset \mathbb{F}[u]$.

Proposition 3.1.8. For an unstable module $M$, the higher derived functors $\Omega_{s}$, $s>1$ of $\Omega$ are trivial, (i.e. $\Omega_{s}=0 \forall s>1$ ) and there is a natural exact sequence in $\mathscr{U}$

$$
0 \rightarrow \Sigma \Omega_{1} M \rightarrow \Phi M \stackrel{\lambda_{M}}{\rightarrow} M \rightarrow \Sigma \Omega M \rightarrow 0
$$


In particular, the complex in $\mathscr{M}$ :

$$
\Sigma^{-1} \Phi M \stackrel{\Sigma^{-1} \lambda_{M}}{\longrightarrow} \Sigma^{-1} M
$$

has homology $\Omega M$ and $\Omega_{1} M$ in homological degrees 0,1 respectively.

Proof. By definition, $\Omega M$ is the destabilization of $\Sigma^{-1} M$. Hence (using the fact that $M$ is unstable),

$$
\Sigma \Omega M \cong M /\left\langle S q^{|x|} x\right\rangle,
$$

which is precisely the cokernel of $\lambda_{M}$.

It is a standard fact that the free unstable modules $F(n)$ are reduced (for example this can be seen since $F(n)$ is a submodule of $H^{*}\left(B V_{n}\right)$, where $V_{n}$ is a rank $n$ elementary abelian 2-group), hence $\lambda_{P}$ is a monomorphism if $P$ is a projective unstable module.

Consider a projective resolution $P_{\bullet} \rightarrow M$ in $\mathscr{U}$. By the above property, the natural transformation $\lambda$ gives rise to a short exact sequence of complexes:

$$
0 \rightarrow \Phi P_{\bullet} \rightarrow P_{\bullet} \rightarrow \Sigma \Omega P_{\bullet} \rightarrow 0 .
$$

The functors $\Phi$ and $\Sigma$ are exact, hence the homology of $P_{\bullet}$ and $\Phi P_{\bullet}$ is concentrated in homological degree zero, where it is respectively $M$ and $\Phi M$, whereas the homology of $\Sigma \Omega P_{\bullet}$ is isomorphic to $\Sigma \Omega_{s} M$ in homological degree $s$, by construction of the derived functors.

The long exact sequence in homology in low degrees gives the exact sequence

$$
0=H_{1}\left(\Phi P_{\bullet}\right) \rightarrow H_{1}\left(\Sigma \Omega P_{\bullet}\right) \rightarrow H_{0}\left(\Phi P_{\bullet}\right)=\Phi M \stackrel{\lambda_{M}}{\rightarrow} M=H_{0}\left(P_{\bullet}\right) \rightarrow H_{0}\left(\Sigma \Omega P_{\bullet}\right),
$$

which shows that the kernel of $\lambda_{M}$ is isomorphic to $\Sigma \Omega_{1} M$, as required.

In higher homological degree, it follows immediately that $\Omega_{s} M=0$ for $s>1$. The final statement is clear.

Corollary 3.1.9. For $p=2$, an unstable module $M$ is reduced if and only if $\Omega_{1} M=0$.

Proof. By definition, $M$ is reduced if and only if $\lambda_{M}$ is injective, hence if and only if $\Sigma \Omega_{1} M=0$. The latter condition is equivalent to $\Omega_{1} M=0$, as required.

\section{Exercise 3.1.10.}

(1) Give an example of a nilpotent unstable module $N$ such that $\Omega N$ is reduced.

(2) Show that $\Omega_{1} N$ is nilpotent if $N$ is a nilpotent unstable module. (Hint: reduce to the case that $N$ is a finitely-generated unstable module and hence to the case that $N$ has a finite filtration with quotients that are suspensions of unstable modules. By induction on the length of the filtration, hence reduce to the case of a suspension.)

(3) Zarati Zar90 showed that an unstable module $M$ (over $\mathbb{F}_{2}$ ) is nil-closed if and only if $M$ and $\Omega M$ are both reduced. Prove this.

Hints:

(a) To show that $\Omega M$ of a nil-closed unstable module is reduced, show that it suffices to consider the nilclosed injective unstable modules and hence reduce to considering the case $M=H^{*}(B V), V$ an elementary abelian 2-group. The rank one case is straightforward; use this together with the behaviour of $\Omega$ on tensor products to treat the general case by induction.

(b) If $M$ is reduced, there is a short exact sequence associated to nil-closure (see Remark 2.3.7):

$$
0 \rightarrow M \rightarrow \bar{M} \rightarrow Q \rightarrow 0,
$$


with $Q$ a nilpotent unstable module and $\bar{M}$ nil-closed. Now use the exact sequence of derived functors of $\Omega$ together with the fact that $\Omega_{1} Q$ is nilpotent.

\subsection{Applications of $\Omega$ and $\Omega_{1}$.}

Proposition 3.2.1. For $C_{\bullet}$ a chain complex of reduced unstable modules, $\Omega C_{\bullet}$ has homology which fits into a natural short exact sequence:

$$
0 \rightarrow \Omega H_{s}\left(C_{\bullet}\right) \rightarrow H_{s}\left(\Omega C_{\bullet}\right) \rightarrow \Omega_{1} H_{s-1}\left(C_{\bullet}\right) \rightarrow 0 .
$$

Proof. Since each $C_{n}$ is reduced, the natural transformation $\lambda$ induces a short exact sequence of complexes

$$
0 \rightarrow \Phi C_{\bullet} \rightarrow C_{\bullet} \rightarrow \Sigma \Omega C_{\bullet} \rightarrow 0 .
$$

Using the exactness of $\Phi$ and $\Sigma$ together with the naturality of $\lambda$, the associated long exact sequence in homology is

$$
\ldots \rightarrow \Phi H_{s}\left(C_{\bullet}\right) \stackrel{\lambda}{\rightarrow} H_{s}\left(C_{\bullet}\right) \rightarrow \Sigma H_{s}\left(\Omega C_{\bullet}\right) \rightarrow \Phi H_{s-1}\left(C_{\bullet}\right) \rightarrow \ldots .
$$

By Proposition 3.1.8, the cokernel of $\lambda_{H_{s}\left(C_{\bullet}\right)}$ is $\Sigma \Omega H_{s}\left(C_{\bullet}\right)$ and its kernel $\Sigma \Omega_{1} H_{s}\left(C_{\bullet}\right)$. Applying these identifications for $s$ and $s-1$ respectively gives the stated short exact sequence.

Corollary 3.2.2. For $s, t \in \mathbb{N}$ and $M$ an unstable module, there is a natural short exact sequence

$$
0 \rightarrow \Omega \Omega_{s}^{t} M \rightarrow \Omega_{s}^{t+1} M \rightarrow \Omega_{1} \Omega_{s-1}^{t} M \rightarrow 0
$$

In particular

$$
\Omega_{s}^{t} M= \begin{cases}0 & s>t \\ \left(\Omega_{1}\right)^{t} M & s=t\end{cases}
$$

Proof. Let $P_{\bullet} \rightarrow M$ be a projective resolution of $M$ in $\mathscr{U}$ and take $C_{\bullet}:=\Omega^{t} P_{\bullet}$, which is a complex of projective unstable modules (by Proposition 2.3.15) and these are reduced. The homology of $C_{\bullet}$ is, by definition, $H_{s}\left(C_{\bullet}\right)=\Omega_{s}^{t} M$, whereas $H_{s}\left(\Omega_{\bullet}\right)=\Omega_{s}^{t+1} M$. The required short exact sequence is furnished by Proposition 3.2 .1 .

The final statement is proved by a straightforward induction upon $t$.

Exercise 3.2.3. Assuming Zarati's theorem that an unstable module $M$ (over $\mathbb{F}_{2}$ ) is nil-closed if and only if $M$ and $\Omega M$ are both reduced, show that $M$ is nilclosed if and only if $\Omega_{s}^{t} M=0$ for $s>0$ and $t \leq 2$.

Remark 3.2.4. For natural numbers $t_{1}, t_{2}$ and an unstable module $M$, there is a Grothendieck spectral sequence

$$
\Omega_{p}^{t_{1}} \Omega_{q}^{t_{2}} M \Rightarrow \Omega_{p+q}^{t_{1}+t_{2}} M
$$

The short exact sequence of Corollary 3.2 .2 corresponds to the case $t_{1}=1$.

Corollary 3.2 .2 leads to an estimation of the connectivity of the modules $\Omega_{s}^{t} M$.

Definition 3.2.5. For $M$ an $\mathscr{A}$-module, the connectivity of $M, \operatorname{conn}(M) \in \mathbb{Z} \cup$ $\{-\infty, \infty\}$, is

$$
\operatorname{conn}(M):=\sup \left\{i \mid M_{j}=0, \forall j \leq i\right\} .
$$

Lemma 3.2.6. For $M$ an $\mathscr{A}$-module, $\operatorname{conn}(\Phi M)=2 \operatorname{conn}(M)+1$.

Proof. An immediate consequence of the definitions of $\Phi$ and of conn.

Proposition 3.2.7. For $s, k \in \mathbb{N}$ and $M$ an unstable module:

$$
\operatorname{conn}\left(\Omega_{s}^{s+k} M\right) \geq 2^{s}(\operatorname{conn}(M)-k) .
$$


Proof. It is clear that conn $(\Omega M) \geq \operatorname{conn}(M)-1$ and, by Lemma 3.2.6, $\operatorname{conn}\left(\Omega_{1} M\right) \geq$ 2 conn $(M)$. The general result is proved by induction upon $s$, using the Grothendieck short exact sequence of Corollary 3.2 .2 for the inductive step.

Remark 3.2.8. Since an unstable module $M$ is always concentrated in non-negative degrees, $\operatorname{conn}(M) \geq-1$, hence it is clear that the previous statement is not optimal.

3.3. Interactions between loops and destabilization. Recall from Proposition 2.3 .17 that, for $t \in \mathbb{N}$, there is a natural isomorphism between $\Omega^{t} D, D \Sigma^{-t}: \mathscr{M} \rightrightarrows$ $\mathscr{U}$. The following result is another application of Proposition 3.2.1

Corollary 3.3.1. For $M$ an $\mathscr{A}$-module, there is a natural short exact sequence:

$$
0 \rightarrow \Omega\left(D_{s} M\right) \rightarrow D_{s}\left(\Sigma^{-1} M\right) \rightarrow \Omega_{1}\left(D_{s-1} M\right) \rightarrow 0 .
$$

Proof. Let $F_{\bullet} \rightarrow M$ be a free resolution of $M\left(\right.$ in $\mathscr{M}$ ) and take $C_{\bullet}=D F_{\bullet}$, which is a complex of projective unstable modules by Proposition 2.3.13

Proposition 2.3.17 implies that $\Omega C_{\bullet}$ is naturally isomorphic to $D\left(\Sigma^{-1} F_{\bullet}\right) ; \Sigma^{-1} F_{\bullet}$ is a projective resolution of $\Sigma^{-1} M$, hence the homology of $\Omega C$. calculates the derived functors $D_{s}\left(\Sigma^{-1} M\right)$, whereas the homology of $C_{\boldsymbol{e}}$ calculates the derived functors $D_{s} M$. The result follows immediately from Proposition 3.2.1.

Remark 3.3.2. The module $\Omega_{1}\left(D_{s-1} M\right)$ is the obstruction to $\Omega\left(D_{s} M\right) \rightarrow D_{s}\left(\Sigma^{-1} M\right)$ being an isomorphism. This is zero if and only if $D_{s-1} M$ is reduced, by Corollary 3.1.9,

Remark 3.3.3. For $m \in \mathbb{N}$ and an $\mathscr{A}$-module $M$, there is a Grothendieck spectral sequence

$$
\Omega_{p}^{m} D_{q} M \Rightarrow D_{p+q} \Sigma^{-m} M .
$$

The short exact sequence of Corollary 3.3.1 corresponds to the case $m=1$.

3.4. Connectivity for $D_{s}$. The explicit identification of the destabilization functor $D M=M / B M$ (see Exercise 2.3.10) leads to the following result:

Lemma 3.4.1. For $M$ an $\mathscr{A}$-module, the natural surjection $M \rightarrow D M$ is an isomorphism in degrees $\leq 2(\operatorname{conn} M+1)$.

Proof. The lowest degree element (if it exists - i.e. if $\operatorname{conn}(M)$ is finite) of $M$ has degree conn $(M)+1$, hence the lowest degree element of $B M$ has degree at least $2(\operatorname{conn}(M)+1)+1$. The result follows.

The following statement is a general result for connected algebras, stated here for the Steenrod algebra.

Lemma 3.4.2. An $\mathscr{A}$-module $M$ has a free resolution $F_{\bullet} \rightarrow M$ in $\mathscr{M}$ with $\operatorname{conn}\left(F_{s}\right) \geq \operatorname{conn}(M)+s$.

Proof. An exercise for the reader.

The following weak result is sufficient for the initial applications; a much stronger result holds (combine Lemma 5.1.6 with Theorem 5.1.8).

Proposition 3.4.3. For $0<s \in \mathbb{N}$ and $M$ an $\mathscr{A}$-module

$$
\operatorname{conn}\left(D_{s} M\right) \geq 2(\operatorname{conn} M+s) .
$$

Proof. It is sufficient to treat the case conn $(M)$ finite (the other cases are clear), hence we may take a free resolution $F_{\bullet} \rightarrow M$ as in Lemma 3.4.2. Consider the 
natural surjection of complexes $F_{\bullet} \rightarrow D F_{\bullet}$. For $s>0$, the portion pertinent to $H_{s}$ is

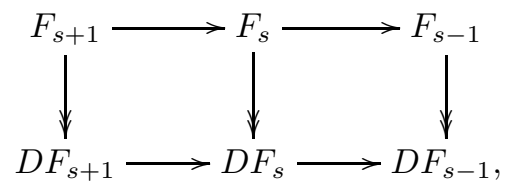

where the top row is exact and the homology of the bottom row (in degree $s$ ) is $D_{s} M$, by definition. The vertical morphisms are all isomorphisms in degrees $\leq 2(\operatorname{conn} M+s)$, by the hypothesis on $F_{\text {}}$ together with Lemma 3.4.1 the result follows.

Notation 3.4.4. For $M$ an $\mathscr{A}$-module and $c \in \mathbb{Z}$, let $M^{>c}$ denote the sub $\mathscr{A}$-module of elements of degree $>c$, so that $\operatorname{conn}\left(M^{>c}\right) \geq c$.

There is a natural short exact sequence of $\mathscr{A}$-modules

$$
0 \rightarrow M^{>c} \rightarrow M \rightarrow M / M^{>c} \rightarrow 0
$$

and natural inclusions $M^{>c+1} \hookrightarrow M^{>c}$ and surjections $M / M^{>c+1} \rightarrow M / M^{>c}$ such that

$$
\begin{aligned}
& M \cong \operatorname{colim}_{c \rightarrow-\infty} M^{>c} \\
& M \cong \lim _{c \rightarrow \infty} M / M^{>c} .
\end{aligned}
$$

Proposition 3.4.5. For $M$ an $\mathscr{A}$-module, $s \in \mathbb{N}$ and $c \in \mathbb{Z}$, the natural morphism

$$
D_{s} M \rightarrow D_{s}\left(M / M^{>c}\right)
$$

is an isomorphism in degrees $\leq 2(c+s-1)$.

Hence the natural morphism

$$
D_{s} M \rightarrow \lim _{c \rightarrow \infty} D_{s}\left(M / M^{>c}\right)
$$

is an isomorphism.

Proof. Consider the long exact sequence for the derived functors $D_{s}$ associated to the short exact sequence (11):

$$
\ldots \rightarrow D_{s}\left(M^{>c}\right) \rightarrow D_{s} M \rightarrow D_{s}\left(M / M^{>c}\right) \rightarrow D_{s-1}\left(M^{>c}\right) \rightarrow \ldots .
$$

Proposition 3.4.3 implies that $\operatorname{conn}\left(D_{s}\left(M^{>c}\right)\right) \geq 2(c+s)$ and $\operatorname{conn}\left(D_{s-1}\left(M^{>c}\right)\right) \geq$ $2(c+s-1)$. The first statement follows immediately, implying the second.

Remark 3.4.6. Proposition 3.4.5 implies that, to study the derived functors $D_{s}$, it is sufficient to consider $\mathscr{A}$-modules $M$ which are bounded above (i.e. such that $M^{>c}=0$ for $\left.c \gg 0\right)$.

3.5. Comparing $D_{s}$ and $\Omega_{s}^{t}$. This section establishes a precise relationship between the derived functors of destabilization and of iterated loop functors. (This material is slightly technical and is not required for the subsequent results, hence can be skipped on first reading.)

Throughout the section, $M$ is taken to be an iterated desuspension of an unstable module, so that there exists $T \in \mathbb{N}$ such that $\Sigma^{t} M$ is unstable $\forall t \geq T$. If $M \neq 0$, conn $(M)$ is finite; by Lemma 3.4.2, there exists a free resolution of $M$ in $\mathscr{M}, F_{\bullet} \rightarrow M$, with $\operatorname{conn}\left(F_{s}\right) \geq \operatorname{conn}(M)+s$. Consider the free resolution $\Sigma^{t} F_{\bullet}$ of $\Sigma^{t} M$, for $t \geq T$. Then, by construction, $D\left(\Sigma^{t} F_{\bullet}\right)$ is a complex of projective unstable modules which has homology $H_{s}\left(D \Sigma^{t} F_{\bullet}\right) \cong D_{s}\left(\Sigma^{t} M\right)$ and, in particular, $H_{0}\left(D \Sigma^{t} F_{\bullet}\right)=\Sigma^{t} M$; Proposition 3.4.3 implies that, for $s>0$, $\operatorname{conn}\left(H_{s}\left(D \Sigma^{t} F_{\bullet}\right)\right)=\operatorname{conn}\left(D_{s}\left(\Sigma^{t} M\right)\right) \geq 2(\operatorname{conn} M+s+t) \geq 2(\operatorname{conn} M+t+1)$. 
Remark 3.5.1. The hypothesis upon $T$ implies that conn $M+T+1 \geq 0$.

The complex $D \Sigma^{t} F_{\bullet}$ can be seen as an approximation to a projective resolution (in unstable modules) of the unstable module $\Sigma^{t} M$. More precisely, one has the following:

Lemma 3.5.2. Let $M$ be an $\mathscr{A}$-module and $t \in \mathbb{N}$ such that $\Sigma^{t} M$ is unstable. There is a short exact sequence of complexes of projectives in $\mathscr{U}$ :

$$
0 \rightarrow D \Sigma^{t} F_{\bullet} \rightarrow P_{\bullet} \rightarrow Q_{\bullet} \rightarrow 0
$$

such that

(1) $P_{\bullet}$ is a projective resolution of $\Sigma^{t} M$ in $\mathscr{U}$;

(2) $D \Sigma^{t} F_{\bullet} \rightarrow P_{\bullet}$ induces an isomorphism on $H_{0}$;

(3) $Q_{0}=0$ and, $\forall s, \operatorname{conn}\left(Q_{s}\right) \geq 2(\operatorname{conn} M+t+1)$.

Proof. (Indications.) This is proved by the algebraic analogue of adding cells in the process of $\mathrm{CW}$ approximation. Starting from the morphism $D \Sigma^{t} F_{\bullet} \rightarrow \Sigma^{t} M$ (considered as a morphism of chain complexes in $\mathscr{U}$ ), one adds 'cells' (free unstable modules) to the complex $D \Sigma^{t} F_{\bullet}$ to obtain a factorization

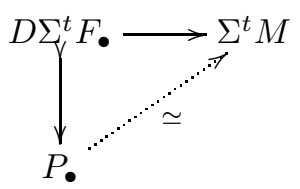

with the dotted map a weak equivalence. By construction, $P_{\bullet}$ is a projective resolution of $\Sigma^{t} M$, the vertical map induces an isomorphism in $H_{0}$, moreover it is an inclusion with cokernel $Q \bullet$ a complex of projective unstable modules.

Finally, the connectivity estimate for the homology of $D \Sigma^{t} F_{\bullet}$ gives a lower bound on the connectivity of the cells which need to be added, hence upon $Q_{\bullet}$.

Proposition 3.5.3. Let $M$ be an $\mathscr{A}$-module and $t \in \mathbb{N}$ such that $\Sigma^{t} M$ is unstable. Then, for all $s \in \mathbb{N}$, the natural morphism

$$
D_{s} M \rightarrow \Omega_{s}^{t} \Sigma^{t} M
$$

is an isomorphism in degrees $\leq 2(\operatorname{conn} M+1)+t$.

Proof. Consider $F_{\bullet} \rightarrow M$ as above and the short exact sequence (2) of Lemma 3.5.2 Applying the functor $\Omega^{t}$ and using the natural isomorphism $\Omega^{t} D\left(\Sigma^{t} F_{\bullet}\right) \cong D F_{\bullet}$ given by Proposition 2.3.17, this yields a short exact sequence of complexes

$$
0 \rightarrow D F_{\bullet} \rightarrow \Omega^{t} P_{\bullet} \rightarrow \Omega^{t} Q_{\bullet} \rightarrow 0,
$$

where the first morphism induces $D_{s} M \rightarrow \Omega_{s}^{t} \Sigma^{t} M$ in homology.

The connectivity condition on $Q_{\bullet}$ implies that $\operatorname{conn}\left(\Omega^{t} Q_{s}\right) \geq 2(\operatorname{conn} M+1)+t$. The result follows from the long exact sequence in homology.

Corollary 3.5.4. For $M$ an $\mathscr{A}$-module and $T \in \mathbb{N}$ such that $\Sigma^{T} M$ is unstable, there is a natural isomorphism

$$
D_{s} M \cong \lim _{T \leq t \rightarrow \infty} \Omega_{s}^{t} \Sigma^{t} M
$$

and the inverse system stabilizes locally for $t \gg 0$ (i.e. in any given degree).

Proof. An exercise for the reader.

Exercise 3.5.5. Let $M \in \mathscr{M}$ be a module which is bounded below $\left(M^{n}=0\right.$ for $n \ll 0)$. Show that, for fixed $s, d \in \mathbb{N}$, there exist $c, T \in \mathbb{N}$ such that $\Sigma^{T}\left(M / M^{>c}\right)$ is unstable and, for all $t \geq T$,

$$
\left(D_{s} M\right)^{d} \cong\left(\Omega_{s}^{t} \Sigma^{t}\left(M / M^{>c}\right)\right)^{d} .
$$




\section{Singer FunCtors}

Singer introduced a series of functors which are indispensable for understanding the derived functors of iterated loops and destabilization. This section recalls the definition of (variants of) these.

4.1. The unstable Singer functors $R_{s}$. Following Lannes and Zarati [LZ87, we recall the construction of the unstable Singer functors $R_{s}$, for $s \in \mathbb{N}$; by convention $R_{0}$ is the identity functor $R_{0}: \mathscr{U} \rightarrow \mathscr{U}$.

Notation 4.1.1. For $s \in \mathbb{N}$, let $D(s)$ denote the $s$ th Dickson algebra, which is defined as the algebra of invariants

$$
D(s):=H^{*}\left(B V_{s}\right)^{G L_{s}},
$$

where the action of the general linear group on the cohomology of the classifying space $B V_{s}$ is induced by the natural action on $V_{s}=\mathbb{F}^{\oplus s}$.

The Dickson algebra $D(s)$ has underlying algebra the polynomial algebra $\mathbb{F}\left[\omega_{s, i} \mid 0 \leq\right.$ $i \leq s-1$ ], where $\omega_{s, i}$ is the Dickson invariant of degree $2^{s}-2^{i}$ (for example, the top Dickson invariant, $\omega_{s, 0}$, is the product of all non-zero classes in $H^{1}\left(B V_{s}\right)$ ). (See Wil83 for further details on the Dickson algebras.)

Notation 4.1.2. For $K$ an unstable algebra, let $K$ - $\mathscr{U}$ denote the category of $K$ modules in $\mathscr{U}$; forgetting the module structure defines a functor $K-\mathscr{U} \rightarrow \mathscr{U}$.

An object of $K-\mathscr{U}$ is an unstable module $M$ equipped with a $K$-module structure such that the structure map $K \otimes M \rightarrow M$ is $\mathscr{A}$-linear.

Proposition 4.1.3. For $K$ an unstable algebra, the category $K$ - $\mathscr{U}$ has a unique abelian structure such that $K-\mathscr{U} \rightarrow \mathscr{U}$ is exact. Moreover the tensor product of $K$-modules $\otimes_{K}$ defines a tensor structure on $K-\mathscr{U}$, with unit $K$ (i.e. $K-\mathscr{U}$ is a symmetric monoidal category $\left.\left(K-\mathscr{U}, \otimes_{K}, K\right)\right)$.

Proof. An unstable algebra $K$ is, in particular, a unital commutative monoid in $\mathscr{U}$ and the category $K-\mathscr{U}$ is its category of modules. The result is standard and is left as an exercise for the reader.

Recall that $H^{*}\left(B V_{1}\right) \cong \mathbb{F}[u]$, with $|u|=1$, has a canonical unstable algebra structure.

Definition 4.1.4. For $M$ an unstable module, let

(1) $S t_{1}: \Phi M \rightarrow \mathbb{F}[u] \otimes M$ denote the linear map (not $\mathscr{A}$-linear) defined by $S t_{1}(x):=\sum u^{|x|-i} \otimes S q^{i}(x)$

(2) $R_{1} M$ denote the sub $\mathbb{F}[u]$-module of $\mathbb{F}[u] \otimes M$ generated by $\left\{S t_{1}(x) \mid x \in M\right\}$.

Remark 4.1.5. The above notation is adopted for typographical simplicity; strictly speaking, $S t_{1}(x)$ should be denoted $S t_{1}(\Phi x)$.

Proposition 4.1.6. [LZ87] For $M$ an unstable module, the sub $\mathbb{F}[u]$-module $R_{1} M \subset$ $\mathbb{F}[u] \otimes M$ is stable under the $\mathscr{A}$-action, hence $R_{1}$ defines a functor $R_{1}: \mathscr{U} \rightarrow$ $\mathbb{F}[u]-\mathscr{U}$.

Proof. The proof is left as an essential exercise for the reader. (Remark 4.1.10 and Exercise 4.1.11 below provide the ingredients. Namely, consider $\Sigma \hat{P} \otimes M$ (using the notation of Remark 4.1.10); Exercise 2.3.10 shows that $B(\Sigma \hat{P} \otimes M)$ is a sub $\mathscr{A}$-module; it suffices to identify this.)

Remark 4.1.7. Forgetting the $\mathbb{F}[u]$-module structure, $R_{1}$ is frequently considered as a functor $R_{1}: \mathscr{U} \rightarrow \mathscr{U}$. However, the $\mathbb{F}[u]$-module structure is important when considering iterated loop functors in Section 5.2 . 
The functor $R_{1}$ has many remarkable properties, such as indicated in Proposition 4.1 .8 below. (The richness of the behaviour of these functors is further exhibited by localizing away from nilpotents; for this, see [Pow12].)

Proposition 4.1.8. (Cf. [LZ87.) Let $M, N$ be unstable modules.

(1) The functor $R_{1}: \mathscr{U} \rightarrow \mathbb{F}[u]-\mathscr{U}$ is exact; more precisely, the underlying $\mathbb{F}[u]$-module of $R_{1} M$ is isomorphic to $\mathbb{F}[u] \otimes \Phi M$.

(2) There is a natural isomorphism of unstable modules

$$
\mathbb{F} \otimes_{\mathbb{F}[u]} R_{1} M \cong \Phi M ;
$$

the canonical surjection is written $\rho_{1}: R_{1} M \rightarrow \Phi M$ and there is a natural short exact sequence in $\mathbb{F}[u]-\mathscr{U}$ :

$$
0 \rightarrow u R_{1} M \rightarrow R_{1} M \rightarrow \Phi M \rightarrow 0
$$

(3) The functor $R_{1}$ preserves tensor products: there is a natural isomorphism $R_{1}(M \otimes N) \cong R_{1} M \otimes_{\mathbb{F}[u]} R_{1} N$.

Proof. See [LZ87] (or prove this as a non-trivial exercise).

\section{Exercise 4.1.9.}

(1) Show that the map $S t_{1}$ is injective and hence deduce part (1) of Proposition 4.1.8.

(2) Show that the projection $\rho_{1}: R_{1} M \rightarrow \Phi M$ is compatible with $\lambda_{M}$, namely the following diagram commutes:

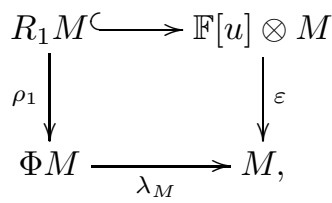

where $\varepsilon$ is induced by the augmentation of $\mathbb{F}[u]$.

(Hint: consider the composite around the top of the diagram, which is a morphism of $\mathbb{F}[u]$-modules.)

(3) Show that the total Steenrod power $S t_{1}$ is multiplicative, when $K$ is an unstable algebra. Namely, for $x, y \in K$,

$$
S t_{1}(x y)=S t_{1}(x) S t_{1}(y)
$$

where the product on the right hand side is formed in the unstable algebra $\mathbb{F}[u] \otimes K$.

(4) For $K$ an unstable algebra, show that $R_{1} K$ is naturally an unstable algebra, equipped with a natural inclusion $\mathbb{F}[u] \hookrightarrow R_{1} K$, so that $R_{1}$ defines a functor $R_{1}: \mathscr{K} \rightarrow \mathbb{F}[u] \downarrow \mathscr{K}$ to the category $\mathbb{F}[u] \downarrow \mathscr{K}$ of $\mathbb{F}[u]$-algebras in $\mathscr{K}$.

(Hint: show that $R_{1} K$ is a sub unstable algebra of $\mathbb{F}[u] \otimes K$. For the morphism $\mathbb{F}[u] \hookrightarrow R_{1} K$, apply $R_{1}$ to the unit $\mathbb{F} \rightarrow K$.)

(5) For $K$ an unstable algebra, show that $R_{1}$ induces a functor $R_{1}: K-\mathscr{U} \rightarrow$ $R_{1} K-\mathscr{U}$.

(Hint: use the functoriality of $R_{1}$.)

(6) Determine the structure of $R_{1} \mathbb{F}\left[u_{2}\right] \subset \mathbb{F}\left[u_{1}, u_{2}\right]$ and identify it as the ring of invariants for the action of the upper triangular subgroup $B_{2} \subset G L_{2}$. (Here $B_{2}$ is isomorphic to the group $\mathbb{Z} / 2$ and acts by $u_{1} \mapsto u_{1}$ and $u_{2} \mapsto u_{2}+u_{1}$. The ring of invariants can be calculated directly.)

Remark 4.1.10. Lannes and Zarati [LZ87] showed that $R_{1}$ is intimately related to destabilization. Namely, the short exact sequence (see Example 2.4.4)

$$
0 \rightarrow \mathbb{F}[u] \rightarrow \hat{P} \rightarrow \Sigma^{-1} \mathbb{F} \rightarrow 0
$$


G. POWELL

defines a non-trivial class $e_{1} \in \operatorname{Ext}_{\mathscr{A}}^{1}\left(\Sigma^{-1} \mathbb{F}, \mathbb{F}[u]\right)$. For an unstable module $M$, tensoring gives the short exact sequence

$$
0 \rightarrow \mathbb{F}[u] \otimes M \rightarrow \hat{P} \otimes M \rightarrow \Sigma^{-1} M \rightarrow 0
$$

and the long exact sequence for derived functors of destabilization induces a morphism

$$
\alpha_{M}: D_{1}\left(\Sigma^{-1} M\right) \rightarrow D(\mathbb{F}[u] \otimes M)=\mathbb{F}[u] \otimes M .
$$

Considering the case $M=\Sigma N$, for an unstable module $N$, Lannes and Zarati observed that $\alpha_{\Sigma N}$ induces a surjection

$$
D_{1} N \rightarrow \Sigma R_{1} N \subset \Sigma \mathbb{F}[u] \otimes N .
$$

In the case $N=\mathbb{F}$, Lannes and Zarati proved moreover that $D_{1} \mathbb{F} \cong \Sigma R_{1} \mathbb{F} \cong$ $\Sigma \mathbb{F}[u]$ (this follows directly from the chain complex constructed in Section 5.1 below). Proposition 3.2 .1 shows that $D_{1}\left(\Sigma^{-1} \mathbb{F}\right) \cong \Omega D_{1} \mathbb{F}$, which is therefore isomorphic to $\mathbb{F}[u]$.

Exercise 4.1.11. (Cf. [LZ87].) Prove the result of Lannes and Zarati stated above that, for $N$ an unstable module,

$$
\alpha_{\Sigma N}: D_{1} N \rightarrow \Sigma R_{1} N \subset \Sigma \mathbb{F}[u] \otimes N
$$

is surjective.

(Hint: using the fact that $N$ is unstable, show that $B(\Sigma \hat{P} \otimes N) \subset \Sigma P \otimes N$ and identifies with $\Sigma R_{1} N \subset \mathbb{F}[u] \otimes \Sigma N$. Here it suffices to consider $S q^{i}\left(\Sigma u^{-1} \otimes x\right)$ for $i>|x|$.)

Remark 4.1.12. The functor $R_{1}$ has topological significance: let $X$ be a pointed topological space and write $E \mathbb{Z} / 2$ for the universal cover of $B \mathbb{Z} / 2$, which is an acyclic space equipped with a free $\mathbb{Z} / 2$-action. (An explicit model is given by $S^{\infty}=$ colim $S^{n}$, with projection $S^{\infty} \rightarrow \mathbb{R} P^{\infty}$ induced by the $\mathbb{Z} / 2$-Galois coverings $S^{n} \rightarrow \underset{n \rightarrow \infty}{R} P^{n}$.)

The diagonal of $X$ induces a $\mathbb{Z} / 2$-equivariant map $E \mathbb{Z} / 2_{+} \wedge X \rightarrow E \mathbb{Z} / 2_{+} \wedge X \wedge X$ (here $(-)_{+}$denotes the addition of a disjoint basepoint) and passage to the quotient by the $\mathbb{Z} / 2$-action gives:

$$
\Delta_{2}: B \mathbb{Z} / 2_{+} \wedge X \rightarrow \mathfrak{S}_{2} X:=E \mathbb{Z} / 2_{+} \wedge_{\mathbb{Z} / 2}(X \wedge X) .
$$

$\mathfrak{S}_{2} X$ is the quadratic construction on the pointed space $X$.

In mod 2 cohomology, this induces

$$
\Delta_{2}^{*}: \tilde{H}^{*}\left(\mathfrak{S}_{2} X\right) \rightarrow H^{*}(B \mathbb{Z} / 2) \otimes \tilde{H}^{*}(X)
$$

and the image of $\Delta_{2}^{*}$ is $R_{1} \tilde{H}^{*}(X)$. This is related to the construction of the Steenrod operations.

The Singer functors can be iterated. For example, $R_{1} R_{1}: \mathscr{U} \rightarrow R_{1} \mathbb{F}[u]-\mathscr{U}$ (see Exercise 4.1.9) and $R_{1} R_{1} M$ is the sub $R_{1} \mathbb{F}[u]$-module of $\mathbb{F}\left[u_{1}, u_{2}\right] \otimes M$ which is generated by $S t_{2}(x):=S t_{1}\left(S t_{1}(x)\right)$.

Notation 4.1.13. For $M$ an unstable module, $s \in \mathbb{N}$ and a fixed basis of $V_{s} \cong \mathbb{F}^{\oplus s}$, define $S t_{s}$ as a linear map

$$
S t_{s}: \Phi^{s} M \rightarrow H^{*}\left(B V_{s}\right) \otimes M
$$

inductively by $S t_{s}=S t_{1} \circ S t_{s-1}$.

Remark 4.1.14. Here, for precision, one should indicate the basis element used for each $S t_{1}$ (cf. [LZ87]). However, this issue is resolved by the following result. 
Lemma 4.1.15. [LZ87] For $M$ an unstable module, the linear map $S t_{s}$ takes values in $D(s) \otimes M \subset H^{*}\left(B V_{s}\right) \otimes M$, hence is independent of the choice of basis of $V_{s}$ used in the definition.

By construction, the iterated Singer functor $R_{1}^{\circ s}$ comes equipped with a natural inclusion $R_{1}^{\circ s} M \hookrightarrow H^{*}\left(B V_{s}\right) \otimes M$, which depends upon the basis used in the construction. This dependency is removed by the following definition:

Definition 4.1.16. For $s \in \mathbb{N}$, let $R_{s}: \mathscr{U} \rightarrow D(s)$ - $\mathscr{U}$ be the functor defined on an unstable module $M$ by

$$
R_{s}(M):=(D(s) \otimes M) \cap R_{1}^{\circ s} M .
$$

Remark 4.1.17. The functor can be defined explicitly by taking $R_{s} M$ to be the sub $D(s)$-module of $D(s) \otimes M$ generated by $S t_{s}(x), \forall x \in M$. The advantage of the previous construction is that it implies immediately that this submodule is stable under $\mathscr{A}$.

Remark 4.1.18. The quadratic nature of the construction is exhibited by the identity for $s \geq 2$ :

$$
R_{s}=\bigcap_{a+b+2=s} R_{1}^{\circ a} R_{2} R_{1}^{\circ b} .
$$

This shows that the functors $R_{s}$ are determined by the generating functor $R_{1}$ and the relation $R_{2} \hookrightarrow R_{1} R_{1}$.

Exercise 4.1.19. Make the previous statement precise and prove it (hint: consider generators for $\left.G L_{s}\right)$.

Exercise 4.1.20. For $0<s \in \mathbb{N}$ and any inclusion $i_{s}: V_{s-1} \subset V_{s}$, show that the canonical inclusions of the Dickson invariants fit into a commutative diagram in $\mathscr{K}:$

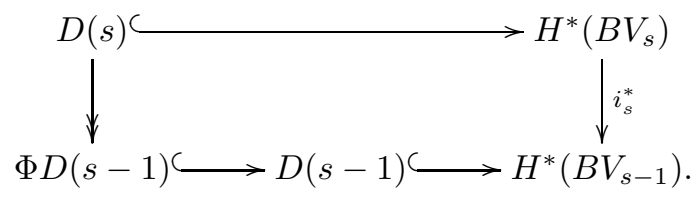

(Use Exercise 3.1 .7 for the first inclusion of the bottom row.) In particular, there is a canonical surjection of unstable algebras $D(s) \rightarrow \Phi D(s-1)$.

Explicitly, show that $i_{s}^{*}$ maps $\omega_{s, 0}$ to zero and $\omega_{s, i} \mapsto \omega_{s-1, i-1}^{2}$ for $i>0$.

Exercise 4.1.21. For $M$ in $D(s-1)-\mathscr{U}$, show that $\Phi M$ is naturally an object of $\Phi D(s-1)-\mathscr{U}$ and hence, via the surjection $D(s) \rightarrow \Phi D(s-1)$, in $D(s)-\mathscr{U}$. (An analogous result holds replacing the module categories such as $D(s)-\mathscr{U}$ by the category $D(s)$ - $\mathscr{M}$ of $D(s)$-modules in $\mathscr{M}$.)

Proposition 4.1.22. For $s \in \mathbb{N}$ and unstable modules $M, N$,

(1) $R_{s}: \mathscr{U} \rightarrow D(s)-\mathscr{U}$ is exact and commutes with tensor products: $R_{s}(M \otimes$ $N) \cong R_{s} M \otimes_{D(s)} R_{s} N$.

(2) The natural transformation $\rho_{1}$ induces a natural surjection $\rho_{s}: R_{s} \rightarrow$ $\Phi R_{s-1}$ via the inclusion $R_{s} \hookrightarrow R_{1} R_{s-1}$ composed with $\left(\rho_{1}\right)_{R_{s-1}}$, which fits into a short exact sequence in $D(s)-\mathscr{U}$ :

$$
0 \rightarrow \omega_{s, 0} R_{s} M \rightarrow R_{s} M \rightarrow \Phi R_{s-1} M \rightarrow 0 .
$$

Proof. See [LZ87] or prove this as an exercise. (Hint: for $M=\mathbb{F}$, the short exact sequence corresponds to the natural projection $D(s) \rightarrow \Phi D(s-1)$.) 
Remark 4.1.23. The class $e_{1} \in \operatorname{Ext}_{\mathscr{A}}^{1}\left(\Sigma^{-1} \mathbb{F}, \mathbb{F}[u]\right)$ of Remark 4.1.10 gives rise, via Yoneda product, to the class $e_{s} \in \operatorname{Ext}_{\mathscr{A}}^{s}\left(\Sigma^{-s} \mathbb{F}, H^{*}\left(B V_{s}\right)\right)$ and it is a fundamental result of Singer's that this class is invariant under the action of $G L_{s}$ (see [LZ87, for example).

Standard methods of homological algebra (it is easier to think in terms of derived categories) show that the functor $D$ induces a natural morphism (for $s \leq t \in \mathbb{N}$ )

$$
\operatorname{Ext}_{\mathscr{A}}^{s}(M, N) \rightarrow \operatorname{Hom}_{\mathscr{U}}\left(D_{t} M, D_{t-s} N\right) .
$$

Thus, the class $e_{s}$ induces a linear morphism (natural in the $\mathscr{A}$-module $M$ )

$$
\alpha_{s}^{M}: D_{s}\left(\Sigma^{-s} M\right) \rightarrow D\left(H^{*}\left(B V_{s}\right) \otimes M\right) .
$$

If $M$ is unstable, the right hand side is $H^{*}\left(B V_{s}\right) \otimes M$ and Lannes and Zarati show that $\alpha_{s}^{M}$ induces a map

$$
\alpha_{s}^{M}: D_{s}\left(\Sigma^{-s} M\right) \rightarrow R_{s} M
$$

(this may also be seen using the results of the next section). This exhibits the relationship between the Singer functor $R_{s}$ and the derived functor of destabilization $D_{s}$.

4.2. Singer functors for $\mathscr{M}$. The unstable Singer functors $R_{s}: \mathscr{U} \rightarrow D(s)-\mathscr{U} \stackrel{\text { forget }}{\longrightarrow}$ $\mathscr{U}$ generalize to

$$
\mathscr{M} \stackrel{R_{s}}{\longrightarrow} D(s)-\mathscr{M} \stackrel{\text { forget }}{\longrightarrow} \mathscr{M},
$$

where $D(s)-\mathscr{M}$ is the category of $D(s)$-modules in $\mathscr{M}$.

Recall that localization gives an inclusion $\mathbb{F}[u] \hookrightarrow \mathbb{F}\left[u^{ \pm 1}\right]$ of $\mathscr{A}$-algebras. If the $\mathscr{A}$-module $M$ is not unstable, then the Steenrod total power $S t_{1}$ (see Definition 4.1.4) on $M$ does not take values in $\mathbb{F}[u] \otimes M$; if $M$ is bounded above it takes values in $\mathbb{F}\left[u^{ \pm 1}\right] \otimes M$ but, in the general case, it is necessary to use a large tensor product $\otimes$ (half-completed tensor product - see [Pow14, for example) so that $S t_{1}$ is a linear map

$$
S t_{1}: \Phi M \rightarrow \mathbb{F}\left[u^{ \pm 1}\right] \underline{\underline{\otimes}} M .
$$

With this modification, $R_{1}$ is defined as in the unstable case, so that $R_{1} M$ comes equipped with a canonical inclusion $R_{1} M \hookrightarrow \mathbb{F}\left[u^{ \pm 1}\right] \underline{\otimes}$. Many of the good properties of $R_{1}$ pass to this setting, in particular:

Proposition 4.2.1. The functor $R_{1}: \mathscr{M} \rightarrow \mathbb{F}[u]-\mathscr{M}$ is exact.

Proof. A generalization of Proposition 4.1.8.

The higher functors $R_{s}$ are constructed as before; the large tensor product leads to some technical issues.

Localization inverting the top Dickson invariant gives a commutative diagram of $\mathscr{A}$-algebras:

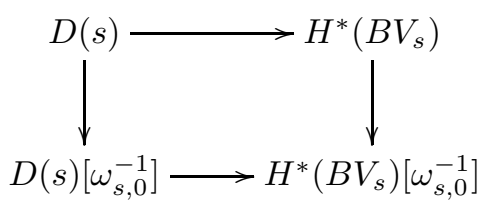

which, in the case $s=1$, corresponds to $\mathbb{F}[u] \hookrightarrow \mathbb{F}\left[u^{ \pm 1}\right]$. The localized Dickson algebra $D(s)\left[\omega_{s, 0}^{-1}\right]$ is the appropriate generalization of $\mathbb{F}\left[u^{ \pm 1}\right]$.

The general Singer functors $R_{s}: \mathscr{M} \rightarrow D(s)$ - $\mathscr{M}$, come equipped with a natural embedding for an $\mathscr{A}$-module $M$

$$
R_{s} M \hookrightarrow D(s)\left[\omega_{s, 0}^{-1}\right] \underline{\underline{\otimes}} M,
$$

and are exact. Moreover, they can be constructed from iterates of $R_{1}$ by imposing the quadratic relation $R_{2}$. 
Remark 4.2.2. Care must be taken in considering the composition because of the large tensor product; see [Pow14] (which is written for the odd characteristic case, but the methods also apply over $\mathbb{F}_{2}$ ).

As in the unstable case, one has the following fundamental short exact sequence:

Proposition 4.2.3. Pow14 For $0<s \in \mathbb{N}$ and $M$ an $\mathscr{A}$-module, there is a natural short exact sequence in $D(s)-\mathscr{M}$ :

$$
0 \rightarrow \Sigma^{-1} R_{s} \Sigma M \rightarrow R_{s} M \rightarrow \Phi R_{s-1} M \rightarrow 0 .
$$

4.3. The Singer differential. There is a new phenomenon when considering the Singer functors defined on $\mathscr{M}$, corresponding to the Singer differential.

Proposition 4.3.1. The residue, namely the unique non-trivial map of graded vector spaces:

$$
\partial: \mathbb{F}\left[u^{ \pm 1}\right] \rightarrow \Sigma^{-1} \mathbb{F},
$$

is $\mathscr{A}$-linear. (Equivalently, $u^{-1}$ is not in the image of $S q^{i}, \forall i>0$ ).

Proof. Since the Steenrod algebra $\mathscr{A}$ is generated by $\left\{S q^{2^{n}} \mid 0<n \in \mathbb{N}\right\}$, it suffices to show that $S q^{2^{n}}\left(u^{-\left(2^{n}+1\right)}\right)=0$ for all $0<n \in \mathbb{N}$. As in Example 2.4.4 the Steenrod total square acts via $S q^{\mathrm{T}}\left(u^{-1}\right)=\frac{u^{-1}}{1+u}$, hence, by multiplicativity of $S q^{\mathrm{T}}$ and using the hypothesis $n>0$,

$$
S q^{\mathrm{T}}\left(u^{-\left(2^{n}+1\right)}\right)=\left(\frac{u^{-1}}{1+u}\right)\left(\frac{u^{-2^{n}}}{1+u^{2^{n}}}\right)=\left(\frac{u^{-1}}{1+u}\right)\left(u^{-2^{n}}+1+\ldots\right) .
$$

It follows that the term in degree -1 is zero, as required.

Definition 4.3.2. For $M$ an $\mathscr{A}$-module, let $d_{M}: R_{1} M \rightarrow \Sigma^{-1} M$ denote the composite natural transformation:

$$
R_{1} M \hookrightarrow \mathbb{F}\left[u^{ \pm 1}\right] \stackrel{\otimes}{=} M \stackrel{\partial \stackrel{\otimes}{=}}{\longrightarrow} \Sigma^{-1} M .
$$

Exercise 4.3.3. Show that, if $M$ is unstable, then $d_{M}: R_{1} M \rightarrow \Sigma^{-1} M$ is trivial.

The following result is the basis for building the chain complex calculating the derived functors of destabilization.

Proposition 4.3.4. For $M$ an $\mathscr{A}$-module, the cokernel of $\Sigma d_{M}: \Sigma R_{1} M \rightarrow M$ is $D M$.

Proof. This is left as a fundamental exercise for the reader.

\section{Constructing Chain complexes}

Recall from Section 4.2 that $R_{s}: \mathscr{M} \rightarrow D(s)$ - $\mathscr{M}$ is an exact functor and that there is a natural differential $d_{M}: R_{1} M \rightarrow \Sigma^{-1} M$ for $M$ an $\mathscr{A}$-module (see Definition 4.3.2). Moreover, there is a natural inclusion $R_{s} \hookrightarrow R_{s-1} R_{1}$. These are the key ingredients to constructing the chain complexes which calculate the derived functors of destabilization and of iterated loop functors.

\subsection{Destabilization.}

Definition 5.1.1. For $M$ an $\mathscr{A}$-module and $1 \leq s \in \mathbb{Z}$, let $d_{s, M}: R_{s} M \rightarrow$ $R_{s-1}\left(\Sigma^{-1} M\right)$ denote the natural morphism given as the composite:

$$
R_{s} M \hookrightarrow R_{s-1} R_{1} M \stackrel{R_{s-1} d_{M}}{\longrightarrow} R_{s-1}\left(\Sigma^{-1} M\right),
$$

so that $d_{1, M}$ identifies with $d_{M}$. 
G. POWELL

Proposition 5.1.2. For $M$ an $\mathscr{A}$-module and $s \in \mathbb{N}$, the composite

$$
R_{s+2}(M) \stackrel{d_{s+2, M}}{\longrightarrow} R_{s+1}\left(\Sigma^{-1} M\right) \stackrel{d_{s+1, \Sigma^{-1} M}}{\longrightarrow} R_{s}\left(\Sigma^{-2} M\right)
$$

is trivial.

Proof. (Indications. See Pow14 for a proof in odd characteristic; the method adapts to $\mathbb{F}_{2}$.) Using the quadratic nature of the functors $R_{s}$, it is straightforward to reduced to the case $s=0$.

This case can be proved using the relationship between the Steenrod algebra and invariant theory, as in the work of Singer Sin83; one method is to embed the diagram in the $\mathbb{F}_{2}$-analogue of the chain complex $\Gamma_{\bullet} M$ considered by Nguyễn $\mathrm{H}$. V. Hưng and Nguyễn Sum [HS95] (their arguments adapt to characteristic two).

Recall that the category of chain complexes for an abelian category is abelian.

Corollary 5.1.3. There is an exact functor $\mathfrak{D}: \mathscr{M} \rightarrow \mathrm{Ch}(\mathscr{M})$ with values in $\mathbb{N}$-graded chain complexes defined on an $\mathscr{A}$-module $M$ by

$$
\begin{aligned}
\mathfrak{D}_{n} M & :=\Sigma R_{s}\left(\Sigma^{s-1} M\right) \\
d_{n}: \mathfrak{D}_{n} M \rightarrow \mathfrak{D}_{n-1} M & :=\Sigma d_{s, \Sigma^{s-1} M} .
\end{aligned}
$$

Proof. Proposition 5.1.2 implies that $\mathfrak{D} M$ is a chain complex and the construction is functorial. Since $R_{s}: \mathscr{M} \rightarrow \mathscr{M}$ is an exact functor (forgetting the action of $D(s)$ ) and $\Sigma$ is exact, the functor $\mathfrak{D}$ is exact.

As shown by the work of Singer on the derived functors of iterated loop functors Sin80, a key input to the proof of the main result is to have a short exact sequence of complexes which gives rise to the long exact sequence of derived functors of destabilization.

Notation 5.1.4. For $M$ an $\mathscr{A}$-module and $s \in \mathbb{N}$, let $\mathbb{D}_{s} M$ denote $H_{s}(\mathfrak{D} \bullet M)$, so that $\mathbb{D}_{0} M=D M$, by Proposition 4.3.4

Proposition 5.1.5. For $M$ an $\mathscr{A}$-module, there is a natural short exact sequence of chain complexes:

$$
0 \rightarrow \Sigma^{-1} \mathfrak{D} \bullet(\Sigma M) \rightarrow \mathfrak{D} \bullet M \rightarrow \Sigma^{-1} \Phi \mathfrak{D} \bullet-1(\Sigma M) \rightarrow 0 .
$$

Moreover, in homology this induces a long exact sequence in $\mathscr{M}$ :

$$
\ldots \rightarrow \Sigma^{-1} \mathbb{D}_{s}(\Sigma M) \rightarrow \mathbb{D}_{s} M \rightarrow \Sigma^{-1} \Phi \mathbb{D}_{s-1}(\Sigma M) \stackrel{\lambda_{s-1}}{\longrightarrow} \Sigma^{-1} \mathbb{D}_{s-1}(\Sigma M) \rightarrow \ldots
$$

The connecting morphism $\lambda_{0}$ identifies with $\Sigma^{-1} \lambda_{D M}$, using the identification $\mathbb{D}_{0} M=$ $D M$.

Proof. Indications. (Cf. Pow14, which treats odd characteristic.) The first statement follows from the naturality of the construction of the chain complex of Proposition 4.2 .3 and of the differential.

For the final statement, the long exact sequence is the long exact sequence in homology, using the exactness of the functors $\Sigma$ and $\Phi$. The identification of $\lambda_{0}$ is straightforward.

Lemma 5.1.6. For $M$ an $\mathscr{A}$-module and $s \in \mathbb{N}$, conn $\left(\mathbb{D}_{s} M\right) \geq 2^{s}(\operatorname{conn} M+s)$.

Proof. Straightforward.

Proposition 5.1.7. $\mathbb{D}_{s}\left(\Sigma^{t} \mathscr{A}\right)=0 \forall t \in \mathbb{Z}$ and $0<s \in \mathbb{N}$. 
Proof. For $s=1$ and $t \in \mathbb{Z}$ recall that $D\left(\Sigma^{t+1} \mathscr{A}\right)=F(t+1)$, so that the long exact sequence of Proposition [5.1.5] is of the following form:

$$
\ldots \rightarrow \Sigma^{-1} \mathbb{D}_{1}\left(\Sigma^{t+1} \mathscr{A}\right) \stackrel{\alpha_{t+1}}{\rightarrow} \mathbb{D}_{1}\left(\Sigma^{t} \mathscr{A}\right) \rightarrow \Sigma^{-1} \Phi F(t+1) \stackrel{\Sigma^{-1} \lambda}{\rightarrow} \Sigma^{-1} F(t+1) \rightarrow \ldots
$$

The morphism $\lambda$ is injective, since $F(t+1)$ is reduced, hence the morphism $\alpha_{t+1}$ is surjective. Since $\operatorname{conn}\left(\mathbb{D}_{1}\left(\Sigma^{t+1} \mathscr{A}\right)\right) \rightarrow \infty$ as $t \rightarrow \infty$, by Lemma 5.1.6, it follows that $\mathbb{D}_{1}\left(\Sigma^{t} \mathscr{A}\right)=0 \forall t \in \mathbb{Z}$.

This forms the initial step of an induction upon $s$; the inductive step is similar (but easier).

Theorem 5.1.8. For $M$ an $\mathscr{A}$-module, there is a natural isomorphism

$$
H_{s}(\mathfrak{D} M) \cong D_{s} M \text {. }
$$

Proof. This follows by standard arguments of homological algebra, since $\mathbb{D}_{0} M=$ $D M$ by Proposition 4.3 .4 and $\mathbb{D}_{s}$ vanishes for $s>0$ on the projectives of $\mathscr{M}$, by Proposition 5.1 .7 .

Remark 5.1.9. From the construction, it is not clear a priori that the homology of the complex should be unstable.

From this result, one recovers immediately one of the main results of Lannes and Zarati [LZ87:

Corollary 5.1.10. For $M$ an unstable module and $s \in \mathbb{N}$, there is a natural isomorphism

$$
D_{s}\left(\Sigma^{1-s} M\right) \cong \Sigma R_{s} M
$$

and a short exact sequence of unstable modules

$$
0 \rightarrow R_{s} M \rightarrow D_{s}\left(\Sigma^{-s} M\right) \rightarrow \Omega_{1} D_{s-1}\left(\Sigma^{1-s} M\right) \rightarrow 0 .
$$

In particular, if $M$ is reduced, then $D_{s}\left(\Sigma^{-s} M\right) \cong R_{s} M$.

Proof. The first statement is a consequence of the vanishing of the relevant differentials in the chain complex $\mathfrak{D} \cdot M$ under the given hypotheses and the second follows from the short exact sequence of Corollary 3.3.1. Finally it is clear that $R_{s} M$ is reduced if $M$ is reduced. Hence, by induction on $s$, one sees that $D_{s}\left(\Sigma^{-s} M\right)$ is reduced and the $\Omega_{1}$ term vanishes.

Remark 5.1.11. Kuhn and McCarty KM13 (who work with homology) give a geometric construction of the analogous chain complex, notably giving a geometric construction of the Singer functors and the differential. The reader should compare the above with their approach, which shows the relationship with the Dyer-Lashof operations.

Exercise 5.1.12. Show that, if $M$ is a finite $\mathscr{A}$-module (of finite total dimension), then the derived functors $D_{s} M$ are all non-trivial for $s \gg 0$.

5.2. Iterated loops. Fix $t \in \mathbb{N}$, which corresponds to the number of loops $\Omega^{t}$.

Notation 5.2.1. For $t \in \mathbb{N}$, let $R_{1 / t}: \mathscr{M} \rightarrow \mathbb{F}[u]-\mathscr{M}$ denote the functor defined on an $\mathscr{A}$-module $M$ by

$$
R_{1 / t} M:=\mathbb{F}[u] /\left(u^{t}\right) \otimes_{\mathbb{F}[u]} R_{1} M,
$$

equipped with the natural projection $R_{1} M \rightarrow R_{1 / t} M$ in $\mathbb{F}[u]-\mathscr{M}$.

Example 5.2.2. For $M$ an $\mathscr{A}$-module, $R_{1 / 0} M=0$ and there is a natural isomorphism $R_{1 / 1} M \cong \Phi M$.

Exercise 5.2.3. Show that

(1) $R_{1 / t}$ is exact; 
(2) $R_{1 / t}$ induces a functor $\mathscr{K} \rightarrow \mathbb{F}[u] /\left(u^{t}\right) \downarrow \mathscr{K}$.

Lemma 5.2.4. For $N$ an unstable module, the differential $d_{\Sigma^{-t} N}$ induces a commutative diagram

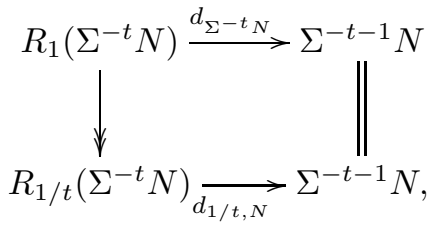

in particular the morphism $d_{1 / t, N}: R_{1 / t}\left(\Sigma^{-t} N\right) \rightarrow \Sigma^{-t-1} N$ is $\mathscr{A}$-linear.

Proof. Straightforward.

The following underlines that the instability hypothesis is essential here:

Exercise 5.2.5. Give an example of an $\mathscr{A}$-module $N$ (necessarily not unstable) for which the diagram is not commutative.

Proposition 5.2.6. For $N$ an unstable module, the cokernel of

$$
\Sigma d_{1 / t, N}: \Sigma R_{1 / t}\left(\Sigma^{-t} N\right) \rightarrow \Sigma^{-t} N
$$

is $\Omega^{t} N$.

Proof. This is left as an important exercise for the reader. (Cf. Proposition 4.3.4.)

Definition 5.2.7. For integers $0 \leq s \leq t$, let $R_{s / t}: \mathscr{M} \rightarrow D(s)$ - $\mathscr{M}$ denote the functor defined on an $\mathscr{A}$-module $M$ by

$$
R_{s / t} M:=\text { image }\left\{R_{s} M \hookrightarrow\left(R_{1}\right)^{\circ s} M \rightarrow R_{1 / t} \circ R_{1 / t-1} \circ \ldots \circ R_{1 / t-s+1} M\right\},
$$

equipped with the canonical surjection $R_{s} M \rightarrow R_{s / t} M$ in $D(s)-\mathscr{M}$.

Remark 5.2.8. The functor $R_{s / t}$ is zero if $s>t$, since $R_{1 / 0}=0$.

Exercise 5.2.9. (This is somewhat harder than some previous exercises.) For integers $0 \leq s \leq t$ and an $\mathscr{A}$-module $M$, describe $R_{1 / t} \circ R_{1 / t-1} \circ \ldots \circ R_{1 / t-s+1} M$ explicitly as a quotient of $\left(R_{1}\right)^{\circ s} M$ by specifying the ideal $I_{s, t} \subset\left(R_{1}\right)^{\circ s} \mathbb{F}$ such that

$$
R_{1 / t} \circ R_{1 / t-1} \circ \ldots \circ R_{1 / t-s+1} M \cong\left(\left(R_{1}\right)^{\circ s} \mathbb{F}\right) / I_{s, t} \otimes_{\left(R_{1}\right)} \circ \mathbb{F}\left(R_{1}\right)^{\circ s} M .
$$

Deduce from this an analogous description of $R_{s / t} M$ in terms of $R_{s} M$.

Proposition 5.2.10. For integers $1 \leq s \leq t$,

(1) $R_{s / t}: \mathscr{M} \rightarrow D(s)-\mathscr{M}$ is exact;

(2) $R_{s / t}$ restricts to an exact functor on unstable modules $R_{s / t}: \mathscr{U} \rightarrow D(s)-\mathscr{U}$.

Proof. Straightforward.

By construction, for an $\mathscr{A}$-module $M$, there is a natural inclusion $R_{s / t} M \hookrightarrow$ $R_{s-1 / t} R_{1 / t-s+1} M$, which fits into the commutative diagram:

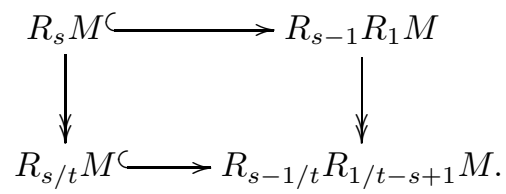


Hence, as in the construction of $d_{1 / t, N}$, there is an induced morphism in $\mathscr{M}$, which is given for $N$ an unstable module by the composite:

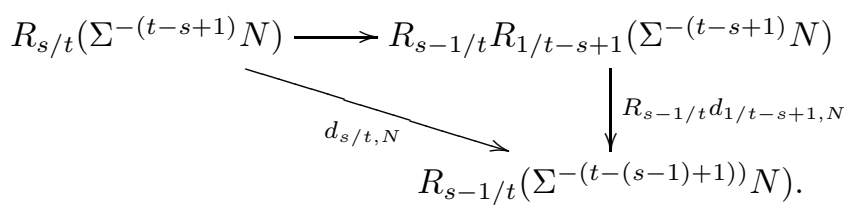

Lemma 5.2.11. For integers $1 \leq s \leq t$ and an unstable module $N$, the following diagram commutes:

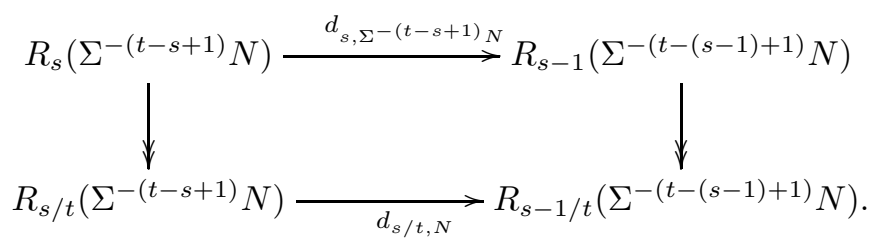

Proof. Straightforward.

Remark 5.2.12. The hypothesis that $N$ be unstable is essential for this compatibility, as in Lemma 5.2.4

There is also an analogue of the short exact sequence of Proposition 4.2.3, based on the observation that, for $t \geq 1$ and $M$ an $\mathscr{A}$-module, the natural surjection $\rho_{1}: R_{1} M \rightarrow \Phi M$ factorizes naturally across a surjection

$$
\rho_{1}: R_{1 / t} M \rightarrow \Phi M \text {. }
$$

Proposition 5.2.13. For integers $1 \leq s \leq t$, the morphism $\rho_{1}$ induces a short exact sequence for the functors $R_{* / t}$ which forms the bottom row of the commutative diagram for $M$ an $\mathscr{A}$-module

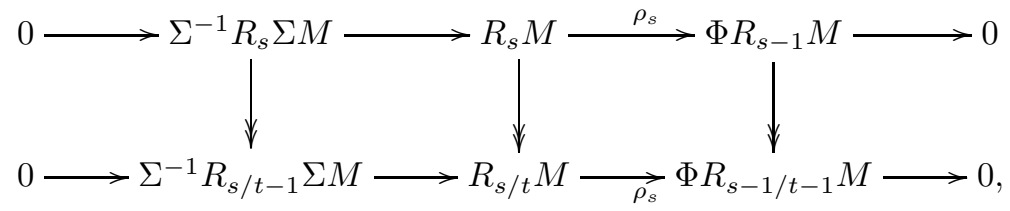

where the top row is provided by Proposition 4.2.3 and the vertical morphisms are the canonical surjections.

Proof. (Indications.) The only non-trivial point is to identify the kernel in the bottom row; this is clear in the case $s=1$ and the higher cases are treated by induction.

Exercise 5.2.14. For $M$ a finite $\mathscr{A}$-module (i.e. the total dimension is finite) and integers $1 \leq s \leq t$,

(1) show that the total dimension of $R_{s / t} M$ is finite;

(2) calculate $\operatorname{conn}\left(R_{s / t} M\right)$ in terms of conn $M$ and $s, t$;

(3) calculate the top dimension of $R_{s / t} M$ in terms of the top dimension of $M$. (Hint: use information on the algebra $R_{s / t} \mathbb{F}$, which can be obtained inductively using Proposition [5.2.13.)

Definition 5.2.15. Let $\mathfrak{C}_{\bullet}^{t}: \mathscr{U} \rightarrow \mathrm{Ch}(\mathscr{M})$ denote the exact functor defined on an unstable module $N$ by $\mathfrak{C}_{s}^{t} N:=\Sigma R_{s / t}\left(\Sigma^{-(t-s+1)} N\right)$ and with differential $\Sigma d_{s / t, N}$.

Remark 5.2.16. The fact that $\mathfrak{C}^{t}$ is a chain complex (namely $d^{2}=0$ ) is a consequence of the corresponding result for $\mathfrak{D}$, which follows from Proposition 5.1.2. 
Exercise 5.2.17. Show that the chain complex $\mathfrak{C}_{\bullet}^{t} N$ is bounded for any unstable module, $N$. Namely, $\mathfrak{C}_{s}^{t} N=0$ for $s>t$.

Proposition 5.2.18. For $N$ an unstable module, there is a natural surjection of chain complexes:

$$
\mathfrak{D}\left(\Sigma^{-t} N\right) \rightarrow \mathfrak{C}^{t}(N) .
$$

Moreover, the short exact sequences of Proposition 5.2.13 induce a short exact sequence of chain complexes which fits into the commutative diagram

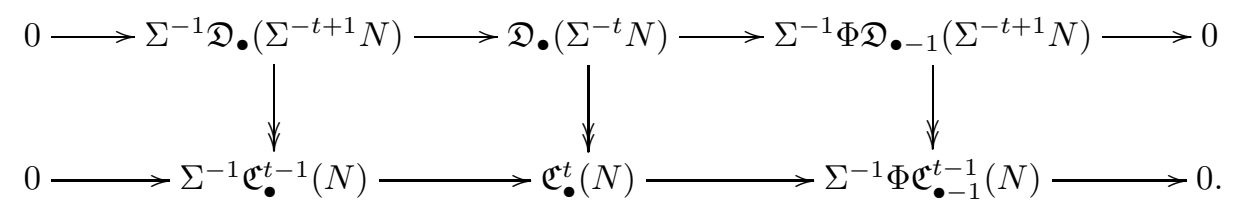

Exercise 5.2.19. Show that $\mathfrak{C}_{\bullet}^{1} N=\left(\Sigma^{-1} \Phi N \rightarrow \Sigma^{-1} N\right)$.

Remark 5.2.20. The functor $N \mapsto \mathfrak{C}_{\bullet}^{t} N$, for $N$ an unstable module, has the same formal properties as that constructed by Singer in Sin80]. The current presentation, being based upon quotients of the Singer functors and the Singer differential, makes explicit the relationship between $\mathfrak{D} \Sigma^{-t}$ and $\mathfrak{C}^{t}$.

Theorem 5.2.21. For $N$ an unstable module and $s, t \in \mathbb{N}$, there is a natural isomorphism

$$
\Omega_{s}^{t} N \cong H_{s}\left(\mathfrak{C}_{\bullet}^{t} N\right)
$$

Moreover, the surjection of chain complexes $\mathfrak{D}_{\bullet}\left(\Sigma^{-t} N\right) \rightarrow \mathfrak{C}_{\bullet}^{t}(N)$ of Proposition 5.2 .18 induces the natural transformations $D_{s}\left(\Sigma^{-t} N\right) \rightarrow \Omega_{s}^{t} N$ in homology.

Proof. (Indications.) The proof of the first point is formally similar to that of Theorem 5.1.8 but the inductive step is easier, since a double induction on $t$ and $s$ can be used. This argument is identical to that used in Singer [Sin80, which only requires the formal properties of the chain complex.

The following result is analogous to Corollary 5.1.10 (and is implicit in [Sin80]).

Corollary 5.2.22. For $N$ an unstable module, $s, t \in \mathbb{N}$ and $k \in \mathbb{N}$ such that $k \geq t-s+1$, there is a natural isomorphism:

$$
\Omega_{s}^{t}\left(\Sigma^{k} N\right) \cong \Sigma R_{s / t}\left(\Sigma^{k-(t-s+1)} N\right)
$$

of unstable modules.

Under these hypotheses, there is a short exact sequence of unstable modules:

$$
0 \rightarrow R_{s / t-1}\left(\Sigma^{k-(t-s+1)} N\right) \rightarrow \Omega_{s}^{t}\left(\Sigma^{k-1} N\right) \rightarrow \Omega_{1} \Omega_{s-1}^{t-1}\left(\Sigma^{k-1} N\right) \rightarrow 0 .
$$

Proof. Straightforward.

Remark 5.2.23. Unlike the functor $R_{1}$, the functor $R_{1 / t}$ restricted to $\mathscr{U}$ does not send reduced unstable modules to reduced objects if $t>1$; in particular, $R_{1 / t} \mathbb{F} \cong$ $\mathbb{F}[u] / u^{t}$ is not reduced for $t>1$.

Example 5.2.24. For $t=1$ and $N$ an unstable module,

(1) for $s=0$ and $k \geq 2, \Omega\left(\Sigma^{k} N\right) \cong \Sigma \Sigma^{k-2} N \cong \Sigma^{k-1} N$, as expected;

(2) for $s=1$, we require $k \geq 1$ and get $\Omega_{1}\left(\Sigma^{k} N\right) \cong \Sigma R_{1 / 1}\left(\Sigma^{k-1} N\right) \cong$ $\Sigma^{-1} \Phi \Sigma^{k} N$, using the identification $R_{1 / 1} \cong \Phi$ and $\Phi \Sigma \cong \Sigma^{2} \Phi$. 
5.3. The Lannes-Zarati homomorphism. The derived functors of destabilization are related to homology over the Steenrod algebra as follows. For $N$ an $\mathscr{A}$-module, there is a natural transformation $D N \rightarrow \mathbb{F} \otimes \mathscr{A} N$ of functors from $\mathscr{A}$-modules to $\mathscr{A}$-modules, where $\mathbb{F} \otimes \mathscr{A} N$ is given the trivial $\mathscr{A}$-module structure. This is obtained by applying the destabilization functor $D$ to the quotient $N \rightarrow \mathbb{F} \otimes_{\mathscr{A}} N$ and then composing with the canonical inclusion:

$$
D N \rightarrow D(\mathbb{F} \otimes \mathscr{A} N)=(\mathbb{F} \otimes \mathscr{A} N)^{\geq 0} \stackrel{\subset}{\longrightarrow} \mathbb{F} \otimes \mathscr{A} N
$$

This passes to derived functors to give

$$
D_{s} N \rightarrow \operatorname{Tor}_{s}^{\mathscr{A}}(\mathbb{F}, N) .
$$

Now, as in Remark 4.1.23 for $M$ an unstable module, there is a natural transformation $\alpha_{s}^{M}: D_{s}\left(\Sigma^{-s} M\right) \rightarrow R_{s} M$. Moreover, by [LZ87, Théorème 2.5], $\alpha_{s}^{\Sigma M}$ induces an isomorphism

$$
D_{s}\left(\Sigma^{1-s} M\right) \stackrel{\cong}{\longrightarrow} \Sigma R_{s} M
$$

Hence there is a natural morphism of $\mathscr{A}$-modules $\Sigma R_{s} M \rightarrow \operatorname{Tor}_{s}^{\mathscr{A}}\left(\mathbb{F}, \Sigma^{1-s} M\right) \cong$ $\Sigma \operatorname{Tor}_{s}^{\mathscr{A}}\left(\mathbb{F}, \Sigma^{-s} M\right)$ and thus $\mathbb{F} \otimes_{\mathscr{A}} R_{s} M \rightarrow \operatorname{Tor}_{s}^{\mathscr{A}}\left(\mathbb{F}, \Sigma^{-s} M\right)$.

The dual of this map,

$$
\operatorname{Ext}_{\mathscr{A}}^{s}\left(\Sigma^{-s} M, \mathbb{F}\right) \rightarrow\left(\mathbb{F} \otimes \mathscr{A} R_{s} M\right)^{*},
$$

is the Lannes-Zarati homomorphism.

Remark 5.3.1. The Lannes-Zarati homomorphism corresponds to an associated graded of the mod 2 Hurewicz map

$$
\pi_{*}\left(\Omega^{\infty} \Sigma^{\infty} X\right) \rightarrow H_{*}\left(\Omega^{\infty} \Sigma^{\infty} X\right)
$$

when $M$ is the reduced cohomology of the pointed space $X$. (The proof of this assertion is sketched in Lan88] and Goe86].)

Singer Sin83] constructed a chain complex $\Gamma_{\bullet}^{+} M$ that computes the homology of $M$ over the Steenrod algebra as a sub-complex of a larger complex $\Gamma \cdot M$. Using the method of Nguyễn H. V. Hưng and Nguyễn Sum [HS95] (adapted to the prime 2) and the material presented here, it is possible to show the following:

Proposition 5.3.2. There is a natural inclusion of chain complexes

$$
\mathfrak{D} \cdot M \hookrightarrow \Gamma_{\bullet}^{+} M
$$

that induces the dual Lannes-Zarati homomorphism in homology.

Remark 5.3.3. Alternative approaches to chain level representations of the LannesZarati homomorphism have been given (see [HT15] for example).

\section{Perspectives}

This section indicates some recent developments and open problems 1 . The ground field is sometimes taken to be $\mathbb{F}_{p}$ with $p$ odd.

\footnotetext{
${ }^{1}$ This material was not presented in the original lectures.
} 
6.1. The spherical class conjecture and related problems. For $X$ a pointed space, the mod 2 Hurewicz map induces

$$
h: \pi_{*}(Q X) \rightarrow H_{*}\left(Q X ; \mathbb{F}_{2}\right),
$$

where $Q X:=\Omega^{\infty} \Sigma^{\infty} X$ is the associated infinite loop space. Here, $\pi_{*}(Q X)$ identifies with the stable homotopy groups $\pi_{*}^{S}(X)$ of $X$.

The famous Curtis conjecture asserts the following:

Conjecture 6.1.1. Cur75 For $X=S^{0}$, the kernel contains all classes except those of odd Hopf or Kervaire invariant.

Various generalizations of the Curtis conjecture have been proposed. Many involve the Adams filtration, which we recall here for a generalized homology theory $E_{*}$ :

Definition 6.1.2. A map $f: X \rightarrow Y$ between spectra has $E$-Adams filtration at least $s \in \mathbb{N}$ if there is a factorization

$$
X=X_{0} \stackrel{f_{0}}{\longrightarrow} X_{1} \stackrel{f_{1}}{\longrightarrow} \ldots \longrightarrow X_{s-1} \stackrel{f_{s-1}}{\longrightarrow} X_{s}=Y
$$

where, for each $i, E \wedge f_{i}$ is null.

Remark 6.1.3. For ordinary cohomology $H \mathbb{F}_{p}$, this corresponds to the filtration arising from the Adams spectral sequence, which has $E_{2}$-page

$$
\operatorname{Ext}_{\mathscr{A}}^{s, t}\left(H^{*}(Y), H^{*}(X)\right)
$$

where the cohomological degree $s$ corresponds to the Adams filtration.

Nguyễn H. V. Hưng has proposed the following generalization of the Curtis conjecture (see HT15]):

Conjecture 6.1.4 (The generalized spherical class conjecture). For $X$ a pointed space, the mod 2 Hurewicz homomorphism

$$
h: \pi_{*}(Q X) \rightarrow H_{*}\left(Q X ; \mathbb{F}_{2}\right)
$$

vanishes on classes of Adams filtration greater than 2.

The Hurewicz map can also be studied in the stable context. Taking $Y$ to be a spectrum and using $\mathbb{F}_{p}$ coefficients, we have the mod $p$ Hurewicz map:

$$
h: \pi_{*}(Y) \rightarrow H_{*}\left(\Omega^{\infty} Y ; \mathbb{F}_{p}\right) .
$$

Motivated by his recent work on the Hurewicz map relating the Adams filtration to a certain augmentation ideal filtration (see Section 6.2 below), Kuhn Kuh14] has proposed:

Conjecture 6.1.5. Let $Y$ be a spectrum such that $H^{*}\left(Y ; \mathbb{F}_{p}\right)$ is finitely generated as an $\mathscr{A}$-module. Then there exists $s$ such that the kernel of

$$
h: \pi_{*}(Y) \rightarrow H_{*}\left(\Omega^{\infty} Y ; \mathbb{F}_{p}\right)
$$

contains all elements of Adams filtration at least $s$.

Remark 6.1.6. If $Y=\Sigma^{\infty} X$, for $X$ a pointed space (satisfying certain finiteness hypotheses), Gaudens and Schwartz GS13 have shown that the hypothesis of Conjecture 6.1 .5 that $H^{*}\left(Y ; \mathbb{F}_{p}\right)$ is finitely generated as an $\mathscr{A}$-module implies that $H^{*}\left(Y ; \mathbb{F}_{p}\right)$ is actually finite.

Hence, in the unstable realm, Conjectures 6.1.4 and 6.1.5 should be considered on finite, pointed CW complexes. Here, the former asserts the stronger form that $s$ can be taken to be 2 . It is possible that the more general stable conjecture proposed by Kuhn could shed further light on the unstable case. 
Remark 6.1.7. The above conjectures are hard. As a first step, it is interesting to consider related algebraic conjectures, as below.

Recall that the Lannes-Zarati homomorphism is an algebraic approximation to the Hurewicz morphism (see Remark 5.3.1). The following was proposed by Nguyễn H. V. Hưng (see [HT15]):

Conjecture 6.1 .8 (The generalized algebraic spherical class conjecture). For $M$ an unstable module, the mod 2 Lannes-Zarati homomorphism

$$
\operatorname{Ext}_{\mathscr{A}}^{s}\left(\Sigma^{-s} M, \mathbb{F}\right) \rightarrow\left(\mathbb{F}_{2} \otimes_{\mathscr{A}} R_{s} M\right)^{*}
$$

vanishes in positive degree for $s>2$.

Remark 6.1.9. This conjecture has been proved for $M=\mathbb{F}_{2}$ and $s \in\{3,4,5\}$ (see Hưn97, Hưn99, Hưn03, HQT14). However, the general case seems to be beyond reach using existing techniques.

A further simplification is obtained by restricting to the image of the algebraic Singer transfer [Sin89]. This leads to the following (cf. [HT15, Conjecture 1.6] and the presentation in [HP16]), in which $R_{s} M$ is considered as a submodule of $P_{s} \otimes M$, where $P_{s}:=H^{*}\left(B V_{s}\right), V_{s}$ a rank $s$ elementary abelian 2-group.

Conjecture 6.1 .10 (The weak generalized algebraic spherical class conjecture). Let $M$ be an unstable $\mathscr{A}$-module (over $\mathbb{F}_{2}$ ) and $s>2$ be an integer. Then every positive degree element of the Singer construction $R_{s} M$ is $\mathscr{A}$-decomposable in $P_{s} \otimes M$.

The full weak generalized algebraic spherical class conjecture was proved as the main result of [HP16]:

Theorem 6.1.11. For $M$ an unstable module (over $\mathbb{F}_{2}$ ) and $2<s \in \mathbb{N}$, the morphism

$$
R_{s} M \rightarrow \mathbb{F}_{2} \otimes_{\mathscr{A}}\left(P_{s} \otimes M\right)
$$

is trivial on elements of positive degree.

Remark 6.1.12.

(1) Theorem 6.1.11 gives evidence supporting Conjecture 6.1.8, in particular providing a result valid for all unstable modules $M$. Theorem 6.1.11 may also lead to further progress on the Conjecture; this is a subject for future research.

(2) Current approaches to Conjecture 6.1.8 rely heavily upon knowledge of the structure of $\operatorname{Ext}_{\mathscr{A}}^{s}\left(\Sigma^{-s} M, \mathbb{F}\right)$, hence are difficult to generalize. One motivation for studying chain-level constructions of the Lannes-Zarati map is to develop methods which do not depend upon such information.

Remark 6.1.13.

(1) Implicit in the above is the relationship between the Singer functors $R_{s}$ and the calculation of the (co)homology of the infinite loop space $Q X$ associated to a pointed space $X$. This relationship is clearer when working in homology, where the Dyer-Lashof operations appear naturally. (The reader is also referred to [Kuh15, Section 2], where a presentation is given which makes use of the Hecke algebra.)

(2) The Singer functors appear naturally in other problems. For example, in Kuh15, Kuhn shows how unstable module theory in conjunction with an understanding of the Singer functors leads to a conceptual, modern proof of the Whitehead Conjecture. 
6.2. Generalizations of the Lannes-Zarati homomorphism. Kuhn Kuh14 has recently provided a new perspective which may allow Lannes-Zarati theory to be extended to certain generalized cohomology theories. Kuhn's constructions rely on working with highly structured commutative ring spectra (here taken to be commutative $S$-algebras, in the sense of [EKMM97]).

Hypothesis 6.2.1. Let $E$ be a commutative $S$-algebra such that $E$ is connective and the unit map induces a surjection $\pi_{0}(S) \rightarrow \pi_{0}(E)$.

Example 6.2.2. The ring spectrum $E$ can be taken to be the $\bmod p$ EilenbergMacLane spectrum $H \mathbb{F}_{p}$, for any prime $p$.

For $X$ a spectrum, the $E$-based unstable Hurewicz map $\pi_{*}(X) \rightarrow E_{*}\left(\Omega^{\infty} X\right)$ is induced by the map of spaces:

$$
h: \Omega^{\infty} X \rightarrow \Omega^{\infty}\left(E \wedge \Sigma^{\infty} \Omega^{\infty} X\right)
$$

which is adjoint to the map $\Sigma^{\infty} \Omega^{\infty} X \rightarrow E \wedge \Sigma^{\infty} \Omega^{\infty} X$ induced by the unit of $E$.

To state Kuhn's result, recall that the E-based Adams resolution of $X$ is the natural decreasing filtration

$$
\ldots \rightarrow X(2) \rightarrow X(1) \rightarrow X(0)=X
$$

where $X(s+1)$ is the fibre of $X(s) \rightarrow E \wedge X(s)$ induced by the unit map of $E$.

Remark 6.2.3. The $E$-based Adams resolution gives another viewpoint on the $E$ Adams filtration introduced in Definition 6.1.2

To illustrate this, observe that the map $X(s) \rightarrow E \wedge X(s)$ admits a retract after smashing with $E$ (using the multiplicative structure of $E$ ), hence

$$
E \wedge(X(s+1) \rightarrow X(s))
$$

is null. In particular, any map of spectra $U \rightarrow X$ that factors across $X(s) \rightarrow X$ has Adams filtration at least $s$.

As explained in [Kuh14, $\Sigma^{\infty} \Omega^{\infty} X$ can be given a natural, non-unital commutative $S$-algebra structure and admits a decreasing augmentation ideal filtration:

$$
\ldots \rightarrow I^{3}(X) \rightarrow I^{2}(X) \rightarrow I(X) \simeq \Sigma^{\infty} \Omega^{\infty} X .
$$

The fundamental new input from $[\mathrm{Kuh14}$ is then the following

Theorem 6.2.4. Suppose that $E$ satisfies Hypothesis 6.2.1 and let $p$ be a prime. Then, localized away from $(p-1)$ !, the Hurewicz map lifts to a map of towers:

$$
h_{s}: \Omega^{\infty} X(s) \rightarrow \Omega^{\infty}\left(E \wedge I^{p^{s}}(X)\right)
$$

that relates the E-based Adams filtration with the augmentation ideal filtration.

Remark 6.2.5. If $X$ is a connective spectrum, there are fibration sequences of spectra for $t \in \mathbb{N}$ :

$$
I^{t+1}(X) \rightarrow I^{t}(X) \rightarrow D_{t} X
$$

where $D_{t} X:=X_{h \mathfrak{S}_{t}}^{\wedge t}$ is the $t$ th extended power construction on the spectrum $X$.

In the case $E=H \mathbb{F}_{p}$, the Singer functors $R_{s}$ (or, rather, their homological counterparts) are closely related to the calculation of the homology $E_{*}\left(D_{p^{s}} X\right)$ in terms of $E_{*}(X)$ (see the construction of the homological Singer functors in KM13, Kuh15] and also [Kuh14]). This establishes the relationship of the above with Lannes-Zarati theory.

Kuhn Kuh14 observes that Lannes-Zarati theory can be generalized when the augmentation ideal filtration is known to split after smashing with $E$. This occurs for example for

(1) $X$ the suspension spectrum $\Sigma^{\infty} Z$ of a space $Z$; 
(2) $E$ the $n$th Morava $E$-theory at the prime $p$, after localizing the filtration with respect to $n$th Morava $K$-theory.

Example 6.2.6. Kuh14 Take $E=H \mathbb{F}_{p}$ and suppose that $Z$ is the suspension of a pointed space. In this case, the homological Singer functors appear as the primitives of the Hopf algebra $H_{*}\left(Q Z ; \mathbb{F}_{p}\right)$, and the $\bmod p$ Hurewicz map induces:

$$
\pi_{*}^{S}(Z) \rightarrow \mathcal{R} H_{*}\left(Z ; \mathbb{F}_{p}\right):=\bigoplus R_{s} H_{*}\left(Z ; \mathbb{F}_{p}\right)
$$

(using homological Singer functors). This is filtration-preserving and recovers LannesZarati's higher Hopf invariants at all primes [LZ84, LZ83.

Example 6.2.7. Let $E$ be the $n$th Morava $E$-theory at the prime $p$. For the technical details involved in applying Theorem 6.2.4, the reader is referred to Kuh14 and, in particular, Kuh14, Corollary 1.17].

It is expected that, when $X$ is a spectrum with $E_{*}(X)$ a finitely-generated free $E_{*}$-module, that there will be an algebraic $E$-theory Lannes-Zarati morphism.

This provides a tantalizing glimpse of higher chromatic analogues of the theory outlined in these notes for singular cohomology, and represents a rich field for future research.

\section{REFERENCES}

[Cur75] Edward B. Curtis, The Dyer-Lashof algebra and the $\Lambda$-algebra, Illinois J. Math. 19 (1975), 231-246. MR 0377885

[EKMM97] A. D. Elmendorf, I. Kriz, M. A. Mandell, and J. P. May, Rings, modules, and algebras in stable homotopy theory, Mathematical Surveys and Monographs, vol. 47, American Mathematical Society, Providence, RI, 1997, With an appendix by M. Cole. MR 1417719

[Goe86] Paul G. Goerss, Unstable projectives and stable Ext: with applications, Proc. London Math. Soc. (3) 53 (1986), no. 3, 539-561. MR 868458 (88d:55011)

[GS13] Gérald Gaudens and Lionel Schwartz, Applications depuis $K(\mathbb{Z} / p, 2)$ et une conjecture de N. Kuhn, Ann. Inst. Fourier (Grenoble) 63 (2013), no. 2, 763-772. MR 3112848

[HM89] J. R. Harper and H. R. Miller, Looping Massey-Peterson towers, Advances in homotopy theory (Cortona, 1988), London Math. Soc. Lecture Note Ser., vol. 139, Cambridge Univ. Press, Cambridge, 1989, pp. 69-86. MR 1055869 (91c:55032)

[HM16] Rune Haugseng and Haynes Miller, On a spectral sequence for the cohomology of infinite loop spaces, Algebr. Geom. Topol. 16 (2016), no. 5, 2911-2947. MR 3572354

[Hưn97] Nguyễn H. V. Hưng, Spherical classes and the algebraic transfer, Trans. Amer. Math. Soc. 349 (1997), no. 10, 3893-3910. MR 1433119 (98e:55020)

[Hưn99] ㄴ The weak conjecture on spherical classes, Math. Z. 231 (1999), no. 4, 727743. MR 1709493

[Hưn03] On triviality of Dickson invariants in the homology of the Steenrod algebra, Math. Proc. Cambridge Philos. Soc. 134 (2003), no. 1, 103-113. MR 1937796

[HP16] Nguyễn H. V. Hưng and Geoffrey Powell, The A-decomposability of the Singer construction, arXiv:1606.09443 (2016).

[HQT14] Nguyễn H. V. Hưng, Võ T. N. Quỳnh, and Ngô A. Tuấn, On the vanishing of the Lannes-Zarati homomorphism, C. R. Math. Acad. Sci. Paris 352 (2014), no. 3, 251254. MR 3167575

[HS95] Nguyễn H. V. Hưng and Nguyên Sum, On Singer's invariant-theoretic description of the lambda algebra: a mod $p$ analogue, J. Pure Appl. Algebra 99 (1995), no. 3, 297-329. MR 1332903 (96c:55024)

[HT15] Nguyễn H. V. Hưng and Ngô A. Tuấn, The generalized algebraic conjecture on spherical classes, preprint 1564 ftp://file.viasm.org/Web/TienAnPham-15/. 2015.

[KM13] Nicholas J. Kuhn and Jason McCarty, The mod 2 homology of infinite loopspaces, Algebr. Geom. Topol. 13 (2013), no. 2, 687-745. MR 3044591

[Kuh14] Nicholas J. Kuhn, Adams filtration and generalized Hurewicz maps for infinite loopspaces, arXiv:1403.7501 (2014).

[Kuh15] - The Whitehead conjecture, the tower of $S^{1}$ conjecture, and Hecke algebras of type A, J. Topol. 8 (2015), no. 1, 118-146. MR 3335250 
[Lan88] J. Lannes, Sur le n-dual du n-ème spectre de Brown-Gitler, Math. Z. 199 (1988), no. 1, 29-42. MR 954749

[Lan92] Jean Lannes, Sur les espaces fonctionnels dont la source est le classifiant d'un pgroupe abélien élémentaire, Inst. Hautes Études Sci. Publ. Math. (1992), no. 75, 135244, With an appendix by Michel Zisman. MR 1179079 (93j:55019)

[LZ83] Jean Lannes and Saïd Zarati, Invariants de Hopf d'ordre supérieur et suite spectrale d'Adams, C. R. Acad. Sci. Paris Sér. I Math. 296 (1983), no. 15, 695-698. MR 705694 (85a:55009)

[LZ84] _ Invariants de Hopf d'ordre supérieur et suite spectrale d'Adams, Preprint, 1984.

[LZ87] Sur les foncteurs dérivés de la déstabilisation, Math. Z. 194 (1987), no. 1, 25-59. MR MR871217 (88j:55014)

[Mar83] H. R. Margolis, Spectra and the Steenrod algebra, North-Holland Mathematical Library, vol. 29, North-Holland Publishing Co., Amsterdam, 1983, Modules over the Steenrod algebra and the stable homotopy category. MR 738973 (86j:55001)

[MM65] John W. Milnor and John C. Moore, On the structure of Hopf algebras, Ann. of Math. (2) 81 (1965), 211-264. MR 0174052 (30 \#4259)

[Mùi75] Huỳnh Mùi, Modular invariant theory and cohomology algebras of symmetric groups, J. Fac. Sci. Univ. Tokyo Sect. IA Math. 22 (1975), no. 3, 319-369. MR 0422451 (54 \#10440)

[Mùi86] C C Chomology operations derived from modular invariants, Math. Z. 193 (1986), no. 1, 151-163. MR 852916 (88e:55015)

[Pow10] Geoffrey M. L. Powell, Module structures and the derived functors of iterated loop functors on unstable modules over the Steenrod algebra, J. Pure Appl. Algebra 214 (2010), no. 8, 1435-1449. MR 2593673

[Pow12] On unstable modules over the Dickson algebras, the Singer functors $R_{s}$ and the functors $\mathrm{Fix}_{s}$, Algebr. Geom. Topol. 12 (2012), 2451-2491 (electronic).

[Pow14] On the derived functors of destabilization at odd primes, Acta Math. Vietnam. 39 (2014), no. 2, 205-236. MR 3212661

[Pri70] Stewart B. Priddy, Koszul resolutions, Trans. Amer. Math. Soc. 152 (1970), 39-60. MR 0265437 (42 \#346)

[Sch94] Lionel Schwartz, Unstable modules over the Steenrod algebra and Sullivan's fixed point set conjecture, Chicago Lectures in Mathematics, University of Chicago Press, Chicago, IL, 1994. MR MR1282727 (95d:55017)

[Sin80] William M. Singer, Iterated loop functors and the homology of the Steenrod algebra. II. A chain complex for $\Omega_{s}^{k} M$, J. Pure Appl. Algebra 16 (1980), no. 1, 85-97. MR MR549706 (81b:55040)

[Sin81] A new chain complex for the homology of the Steenrod algebra, Math. Proc. Cambridge Philos. Soc. 90 (1981), no. 2, 279-292. MR MR620738 (82k:55018)

[Sin83] , Invariant theory and the lambda algebra, Trans. Amer. Math. Soc. 280 (1983), no. 2, 673-693. MR MR716844 (85e:55029)

[Sin89] The transfer in homological algebra, Math. Z. 202 (1989), no. 4, 493-523. MR 1022818 (90i:55035)

[Sin78] Iterated loop functors and the homology of the Steenrod algebra, J. Pure Appl. Algebra 11 (1977/78), no. 1-3, 83-101. MR MR0478155 (57 \#17644)

[Wil83] Clarence Wilkerson, A primer on the Dickson invariants, Proceedings of the Northwestern Homotopy Theory Conference (Evanston, Ill., 1982), Contemp. Math., vol. 19, Amer. Math. Soc., Providence, RI, 1983, pp. 421-434. MR 711066 (85c:55017)

[Zar84] Saïd Zarati, Dérivés du foncteur de déstabilisation en caractéristique impaire et applications, Thèse d'état, Université Paris Sud, 1984.

[Zar90] Derived functors of the destabilization and the Adams spectral sequence, Astérisque (1990), no. 191, 8, 285-298, International Conference on Homotopy Theory (Marseille-Luminy, 1988). MR MR1098976 (92c:55020)

LABORATOIRE ANGEVIN DE RECHERCHES EN MATHÉmATIQUES (LAREMA), CNRS, Université D'Angers, Université Bretagne Loire, 2 Bd lavoisier 49045 Angers Cedex 01

E-mail address: Geoffrey.Powell@math.cnrs.fr 